\title{
L'exploitation alimentaire et technique du gibier au début du Paléolithique supérieur aux Abeilles (Haute-Garonne, France)
}

Food and technical exploitation of mammals during the early Upper Palaeolithic at Les Abeilles (Haute-Garonne, France)

\section{Marie-Cécile Soulier}

\section{OpenEdition}

Journals

Édition électronique

URL : http://journals.openedition.org/paleo/2829

DOI : $10.4000 /$ paleo.2829

ISSN : 2101-0420

Éditeur

SAMRA

Édition imprimée

Date de publication : 28 décembre 2014

Pagination : 287-307

ISSN : 1145-3370

Référence électronique

Marie-Cécile Soulier, «L'exploitation alimentaire et technique du gibier au début du Paléolithique supérieur aux Abeilles (Haute-Garonne, France) », PALEO [En ligne], 25 | 2014, mis en ligne le 28 juillet 2015, consulté le 07 juillet 2020. URL : http://journals.openedition.org/paleo/2829 ; DOI : https:// doi.org/10.4000/paleo.2829

\section{(2) $\odot \Theta \Theta$}

PALEO est mis à disposition selon les termes de la licence Creative Commons Attribution - Pas d'Utilisation Commerciale - Pas de Modification 4.0 International. 


\title{
L'exploitation alimentaire et technique du gibier au début du Paléolithique supérieur aux Abeilles (Haute-Garonne, France)
}

\author{
Marie-Cécile SOULIER(1)
}

Résumé : La grotte des Abeilles, fouillée entre 1945 et 1951, a livré des niveaux archéologiques documentant notamment les premières phases du Paléolithique supérieur en Europe. Ces séries, attribuées au Protoaurignacien et à l'Aurignacien ancien, se composent de plusieurs restes humains, d'un abondant matériel lithique et de nombreux vestiges fauniques dont certains ont été transformés en outils et en éléments de parure. L'étude présentée ici concerne l'ensemble du matériel faunique, soit les restes utilisés à des fins alimentaires, techniques et ornementales. Les analyses archéozoologiques des niveaux attribués au Protoaurignacien et à l'Aurignacien ancien illustrent à la fois des similitudes (saisons de capture du gibier, modes de transport des carcasses) et des différences (e.g. désarticulation, techniques de récupération de la moelle osseuse) dans les modalités d'exploitation alimentaire du gibier. La prise en compte de l'ensemble du matériel faunique a permis de montrer que les sphères alimentaire, utilitaire et symbolique s'enchevêtrent de manière complexe dans les deux ensembles et que les choix effectués se répercutent sur l'ensemble de la chaîne d'exploitation (acquisition du gibier, modalités de boucherie, etc.). Les données acquises, confrontées à celles disponibles pour d'autres séries de cette même période, soulignent la diversité des réponses adoptées par les groupes humains du début du Paléolithique supérieur pour satisfaire au mieux à des besoins spécifiques.

Mots-clés : Paléolithique supérieur, archéozoologie, Protoaurignacien, Aurignacien ancien, Sud-Ouest de la France, modalités de boucherie, exploitation utilitaire-symbolique de la faune, chaîne opératoire d'exploitation de la faune.

\begin{abstract}
Food and technical exploitation of mammals during the early Upper Palaeolithic at Les Abeilles (Haute-Garonne, France). The cave of Les Abeilles, which was excavated from 1945-1951, comprises archaeological layers that document the early phases of the Upper Palaeolithic in Europe. The Protoaurignacian and Early Aurignacian layers contain several human remains, as well as an abundant lithic material. A large number of faunal remains have been uncovered in these layers, including some that were transformed into tools and ornaments. The present study is concerned with the entire faunal material, including food debris and remains used for technical or ornamental purposes. The archaeozoological analysis of the Protoaurignacian and Early Aurignacian layers show similarities (hunting seasons, carcass transport decisions) as well as differences (e.g., dismembering, marrow processing) in carcass processing. By examining the entire faunal material, this study shows that the dietary, technical and symbolic spheres are strongly interconnected in both layers, and this in a complex way. The decisions that were made influenced the entire processing sequence (prey acquisition, butchery processes, etc.). Comparisons of these data with coeval assemblages highlight the fact that early Upper Palaeolithic groups used a wide range of responses to fulfill their particular needs.
\end{abstract}

Key-words: Upper Palaeolithic, zooarchaeology, Protoaurignacian, Early Aurignacian, southwestern France, carcass processing, domestic and symbolic use of the fauna, sequence of exploitation of animals.

(1)Trent University, Dept. of Anthropology, DNA Block C, 2140 East Bank Drive, Peterborough, ON, K9J 7B8 Canada et Université de Toulouse-Le Mirail TRACES UMR 5608 CNRS, Maison de la Recherche, 5 allées Antonio Machado, FR-31058 Toulouse Cedex 9 mariecsoulier@gmail.com 


\section{1 - Introduction}

En Europe occidentale, le début du Paléolithique supérieur est marqué par de profondes mutations au sein des industries dont certaines sont directement liées à l'exploitation du gibier. En effet, de nouvelles armes de chasse apparaissent (e.g. Pelegrin 1990 ; Bon 2002 ; Bordes 2002 ; Normand 2005 ; Teyssandier 2007 ; Bachellerie 2011 ; Roussel 2011) et l'animal devient une source de matière première pour la réalisation d'outils et de parures (e.g. Leroy-Prost 1974, 1975, 1979 ; Taborin 1990, 2002 ; Knecht 1991, 1993 ; Liolios 1999, 2006 ; Julien, Baffier, Liolios 2002 ; Vanhaeren 2002 ; White 2002, 2010 ; Tartar 2009 ; Soulier et al. 2014). Les études archéozoologiques restent toutefois rares pour les phases initiales du Paléolithique supérieur (Sekhr 1998 ; Letourneux 2003 ; Castel 2011 ; Soulier 2013), ce qui est largement imputable aux nombreuses limites du corpus disponible (problèmes taphonomiques, ancienneté des opérations de fouilles).

Le site des Abeilles (Montmaurin, Haute-Garonne) emblématique car à l'origine de la reconnaissance du Protoaurignacien (Laplace 1966) - a livré des niveaux archéologiques attribués au Protoaurignacien et à l'Aurignacien ancien. Les industries lithiques ont été réanalysées récemment (Eizenberg 2006 ; Bon comm. pers. 2011) et les restes fauniques récoltés, abondants, correspondent à la fois à des déchets alimentaires et à des pièces transformées. Le matériel faunique des Abeilles offre donc l'opportunité de mieux appréhender les modalités d'exploitation du gibier à cette période charnière de la Préhistoire.

Grâce à une étude archéozoologique incluant l'ensemble des restes fauniques (restes issus de l'alimentation, industrie en matière dure animale et parure), cet article vise à documenter les modalités d'exploitation de la faune opérées par les groupes humains qui ont occupé le site des Abeilles au début du Paléolithique supérieur. La confrontation des données issues des ensembles attribués au Protoaurignacien et à l'Aurignacien ancien permet ainsi de discuter d'éventuelles différences dans les modalités d'exploitation alimentaire du gibier au cours du Protoaurignacien et de l'Aurignacien ancien. La prise en compte de l'ensemble des restes de faune permet par ailleurs de s'interroger sur les liens existants entre les sphères techniques et ornementales et la chaîne d'exploitation alimentaire.

\section{2 - Présentation générale du gisement}

La grotte des Abeilles (fig. 1a) est située dans le massif calcaire de Lespugue-Montmaurin en Haute-Garonne (Méroc 1963). Si le gisement s'inscrit dans un environnement de faibles altitudes et de reliefs collinaires, la grotte s'ouvre toutefois dans les reliefs abrupts des gorges de la Seygouade. La grotte est orientée sud-ouest et correspond à une galerie qui se subdivise en deux salles (fig. 1b).

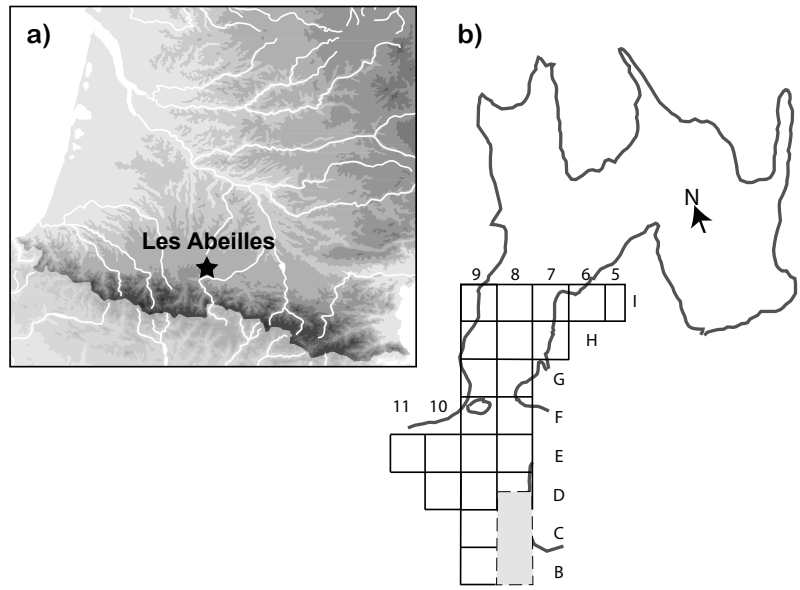

Figure 1 - a) Localisation du gisement des Abeilles et b) plan de la grotte avec carroyage de la zone fouillée (modifié d'après Laplace et al. 2006). Le second sondage est matérialisé en grisé.

Figure 1 - a) Location of Les Abeilles and b) layout of the cave and excavation grid (modified from Laplace et al. 2006). The second test pit is shown in grey.

Deux sondages - l'un en 1945 et l'autre en 1947 (Méroc 1948) - ont été effectués par R. Cammas, l'inventeur du site. La grotte a ensuite été fouillée entre 1948 et 1951 sur une surface d'environ $22 \mathrm{~m}^{2}$, au niveau de l'entrée et à l'intérieur de la cavité. Une stratigraphie de près d'1,50 m de puissance a été mise au jour (fig. 2), au sein de laquelle trois techno-complexes ont été distingués (Laplace et al. 2006) : Moustérien (c. 4), Protoaurignacien (c. 3 et 2) et Aurignacien ancien (c. 1). Une analyse récente de la stratigraphie et des carnets de terrain a mis en évidence les difficultés qu'ont eues les fouilleurs à reconnaître le Protoaurignacien lors des premiers temps de la fouille (Eizenberg 2006). Bien qu'il existe deux carnets de terrain (dont un quasiment vierge), les méthodes de fouilles ne sont pas détaillées. On sait néanmoins qu'un carroyage a été installé. L. Méroc fouillait les grottes de Montmaurin au même moment, ce qui a permis à $R$. Cammas de se familiariser avec la méthode de fouille basée sur l'application des coordonnées cartésiennes (Laplace et Méroc 1954). Dans cette méthode, le tamisage est préconisé (Laplace 1971). Si les carnets de terrain ne mentionnent pas cette pratique, la présence de nombreuses pièces lithiques de petites dimensions suggère qu'au moins certaines zones ont été tamisées (Eizenberg 2006).

Des dents humaines ont été récoltées dans les deux ensembles aurignaciens mais aucune ne permet d'attribution spécifique (Gambier 1992).

L'abondant matériel lithique mis au jour aux Abeilles a initialement été analysé par G. Laplace (1966), matériel qui lui a notamment permis de définir pour la première fois le Protoaurignacien. Le matériel lithique de la c. 2 (Protoaurignacien) a récemment fait l'objet d'une étude 


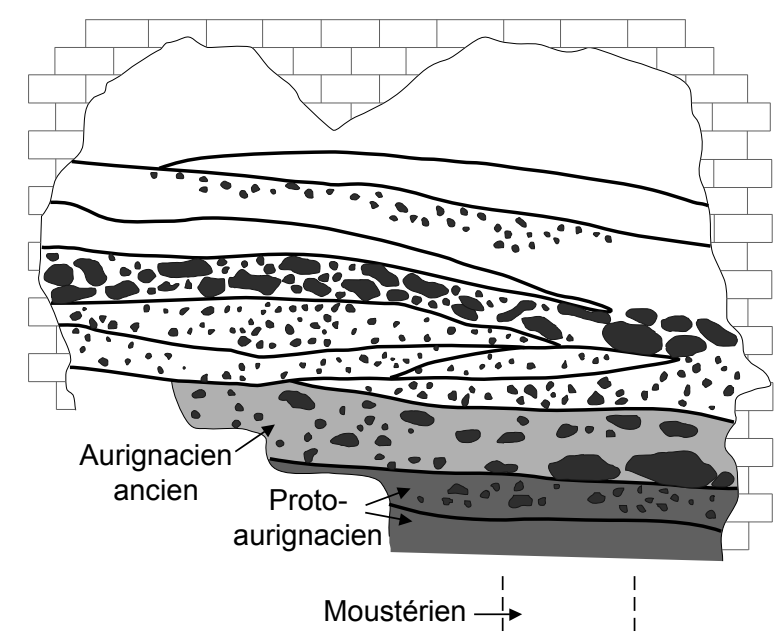

Figure 2 - Coupe frontale de la grotte des Abeilles (modifié d'après Laplace et al. 2006).

Figure 2 - Frontal profile of the cave of Les Abeilles (modified from Laplace et al. 2006).

typo-technologique détaillée (Eizenberg 2006) et un diagnostique de la c. 1 (Aurignacien ancien) a été effectué (F. Bon comm. pers 2011). Ces deux études documentent un outillage essentiellement confectionné en matière première locale, provenant de la vallée de la Seygouade (Simonnet 1979). Cette matière première a été utilisée pour produire des outils expédients et peu retouchés (Eizenberg 2006 ; Bon comm. pers. 2011). De rares outils dont la matière première provient de gîtes éloignés (e.g. Chalosse, Bidache de type Salies) témoignent d'un plus fort investissement technique et paraissent avoir été importés sous forme de produits finis (op. cit.). Outre les différences classiquement mises en avant dans les schémas de débitage entre Protoaurignacien et Aurignacien ancien (e.g. Bon 2002 ; Bordes 2002 ; Teyssandier 2007), les deux ensembles se distinguent en termes de conservation, avec un niveau Protoaurignacien fortement affecté par la retouche mécanique (Eizenberg 2006) qui contraste avec les états de surfaces beaucoup plus frais du niveau Aurignacien ancien (Bon comm. pers. 2011).

Plusieurs pièces d'industrie en matière dure animale et de parure ont été mises au jour dans le gisement des Abeilles et ont été décrites par I. Barandiáran (2006). Cinq pièces ont été récoltées dans le niveau attribué au Protoaurignacien : deux probables lissoirs, un objet plat, une sagaie à base fendue et une pendeloque rectangulaire en os. L'ensemble attribué à l'Aurignacien ancien a livré 48 pièces d'industrie en matière dure animale et se compose de six sagaies à base fendue retrouvées pratiquement complètes (et deux fragments), de pointes en bois de cervidé et en os, de lissoirs, d'une " esquille aiguisée en os ", de deux retouchoirs et d'un fragment d'ivoire décrit comme probable fragment de bâton percé. Plusieurs déchets de débitage sont présents, et notamment ceux liés à l'extraction de baguettes sur bois de cervidés. Deux pendeloques sur canines de carnivores de taille moyenne, une sur incisive d'herbivore de taille moyenne et deux plaquettes perforées en ivoire ont également été décrites (Barandiáran 2006).

Les restes fauniques ont fait l'objet d'une première détermination anatomique et spécifique par J. Altuna (2006). Cette étude, qui porte sur 294 restes pour l'ensemble attribué au Protoaurignacien et sur 477 pour l'Aurignacien ancien, indique que les ongulés et les carnivores sont présents en proportions équivalentes dans les deux ensembles. Dans cette étude, le trio RenneCheval-Bovinés constitue la majorité des ongulés, et est associé au Cerf, au Bouquetin, à l'Isard, au Sanglier et, dans la c. 1, potentiellement au Mégacéros. Les Carnivores les plus fréquents sont le Renard, l'Ours et, dans une moindre mesure, l'Hyène. L'Ours est particulièrement abondant dans l'ensemble Protoaurignacien puisqu'il participe à hauteur de $22,4 \%$ au spectre faunique ; le Renard représente $24,9 \%$ des pièces déterminées dans l'Aurignacien ancien.

\section{3 - Présentation des méthodes d'analyse et du corpus}

La détermination anatomique et spécifique a été réalisée à l'aide de la collection de référence du Musée National de Préhistoire des Eyzies-de-Tayac. Les pièces n'ayant pu être déterminées précisément ont été attribuées à des classes de taille. Le Nombre de Restes (NR) ${ }^{2}$, le Nombre de Restes total (NRt), le Nombre de Restes déterminés (NRd), les Unités Animales Minimum (MAU) et le Nombre Minimum d'Individus (NMI) ont été calculés. Le calcul du NMI prend en compte les données d'âge et de latéralisation. Chaque reste déterminable ou supérieur à $2 \mathrm{~cm}$ a été observé à la loupe (grossissement x30) ; les pièces de dimensions inférieures ont uniquement fait l'objet d'une observation à l'œil nu. Les traces climatoédaphiques, de manducation et celles liées à une activité anthropique (e.g. Fischer 1995) ont été notées. L'analyse des bords de fracture a été effectuée d'après les critères proposés par Villa et Mahieu (1991). L'âge au décès des proies a été estimé à partir des séquences d'usure et d'éruption dentaire (Miller 1974 ; Frison, Wilson M., Wilson D.J. 1976 ; Levine 1979 ; Bignon 2006). Les saisons de capture ont été évaluées à partir de l'observation des dents déciduales (Miller 1974 ; Brugal et David 1993 ; Bignon 2006) et des os de fœtus (Habermehl 1975 ; Roine, Nieminen, Timisjävi 1982). Les représentations squelettiques ont été confrontées - via des tests de corrélation de Spearman $\left(r_{s}\right)$ - à la densité des portions des différents ossements (d'après Kreutzer 1992 ; Lam et al. 1999 ) et aux indices d'utilité nutritive (Metcalfe et Jones 1988 ; Emerson 1990 ; Outram et Rowley-Conwy 1998 ; Morin 2007). La quantification des stries de découpe s'est faite à partir du NR avec surface observable (NRo)

(2) Pour un fragment de mandibule chaussée de plusieurs dents, le NR a été noté comme égal à 1 . 
uniquement et inclut les restes dentaires. Les actions à l'origine des stries de boucherie ont été interprétées via la compilation de référentiels de boucherie expérimentaux et ethnographiques (Binford 1981 ; Bez 1995 ; Nilssen 2000 ; Vigne 2005 ; Costamagno et David 2009 ; Thiébaut et al. $2011)^{3}$. La quantification des encoches de percussion s'est, elle, faite uniquement à partir du NRt d'os à cavité médullaire (mandibule, humérus, radio-ulnaire, fémur, tibia, os longs indéterminés, os du métapode, première et deuxième phalanges). Les os brûlés ont été classés par degré de combustion, taille et type de tissu afin de déterminer leur origine (accidentelle, combustible, etc. : Costamagno et al. 2009). Les « brûlures distales » (traces de chauffe légères situées aux extrémités des os : e.g. Vigne et al. 1981) ont également été notées.

Le matériel faunique présenté ici correspond au Protoaurignacien (c. 2 et 3 ) et à l'Aurignacien ancien (c. 1). Les c. 2 et 3 ont été regroupées pour l'étude car la c. 3 correspondrait à la base de la c. 2 (Laplace et al. 2006). En raison des problèmes de reconnaissance des couches protoaurignaciennes au début des fouilles (Eizenberg 2006), certains carrés ont été exclus de l'analyse (détails dans Soulier 2013). Au total, 2561 vestiges fauniques ont été étudiés pour l'ensemble attribué au Protoaurignacien et 12281 pour le niveau Aurignacien ancien. Ces pièces correspondent aux déchets alimentaires, aux pièces d'industrie en matière dure animale et aux éléments de parure.

\section{4 - Résultats}

\section{1 - Taphonomie}

Les deux ensembles sont fortement fragmentés, avec plus de la moitié des restes mesurant moins de $2,5 \mathrm{~cm}$ (fig. $3 a$ ). La présence de pièces inférieures à $1 \mathrm{~cm}$ appuie, dans une certaine mesure, l'hypothèse d'une fouille avec tamisage. Les os à cavité médullaire complets sont rares et l'aspect des bords de fracture indique que cette fragmentation s'est essentiellement produite sur os frais (fig. 3b). Aucun remontage inter-ensemble n'a été identifié ; lorsque les indications de provenance sont disponibles, les regroupements concernent systématiquement des pièces issues d'un même carré (Soulier 2013).

La majorité des surfaces osseuses sont exemptes de toute altération dans les deux ensembles (58\% dans l'ensemble attribué au Protoaurignacien et $57 \%$ pour l'Aurignacien ancien). En raison de nombreuses pièces abrasées, $30 \%$ des surfaces osseuses de l'ensemble attribué au Protoaurignacien sont illisibles, contre seulement $10 \%$ pour l'Aurignacien ancien. L'analyse statistique des traces présentes sur les restes fauniques identifie des différences hautement significatives entre les deux niveaux pour toutes les altérations testées à l'exception des fissures (tabl. 1). Les surfaces osseuses du niveau Protoaurignacien sont couramment abrasées -ce qui fait écho aux observations sur le matériel lithique (cf. supra)- tandis que celles du niveau Aurignacien ancien portent fréquemment des traces de racines. L'ensemble attribué au Protoaurignacien est

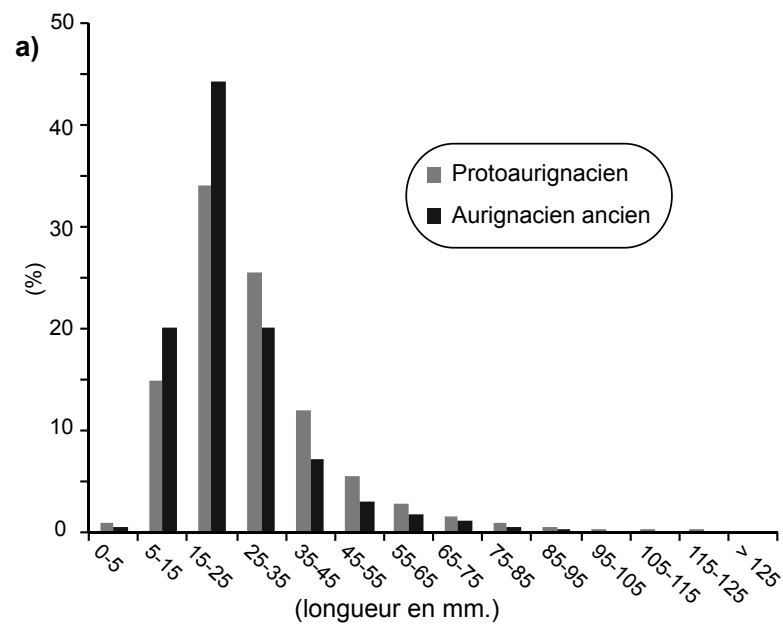

b)

\begin{tabular}{|c|c|c|c|c|c|}
\hline & \multicolumn{2}{|c|}{ Protoaurignacien } & \multicolumn{2}{|c|}{ Aurignacien ancien } \\
\hline & & NR & $\%$ & NR & $\%$ \\
\hline \multirow{5}{*}{ longueur } & 1 & 526 & 88,4 & 1132 & 84,2 \\
\hline & 2 & 46 & 7,7 & 133 & 9,9 \\
\hline & 3 & 11 & 1,8 & 59 & 4,4 \\
\hline & 4 & 8 & 1,3 & 11 & 0,8 \\
\hline & 5 & 4 & 0,7 & 9 & 0,7 \\
\hline \multirow{3}{*}{ circonférence } & 1 & 575 & 96,6 & 1288 & 95,8 \\
\hline & 2 & 9 & 1,5 & 22 & 1,6 \\
\hline & 3 & 11 & 1,8 & 34 & 2,5 \\
\hline \multirow{4}{*}{$\begin{array}{l}\text { Type de } \\
\text { fracture }\end{array}$} & frais & 296 & 78,9 & 1532 & 83,7 \\
\hline & $\mathrm{sec}$ & 66 & 17,6 & 105 & 5,7 \\
\hline & feu & 0 & 0,0 & 18 & 1,0 \\
\hline & récent & 13 & 3,5 & 175 & 9,6 \\
\hline
\end{tabular}

Figure 3 - a) Longueur en $\mathrm{mm}$ des restes osseux récoltés aux Abeilles et b) description des os à cavité médullaire : longueur et circonférence conservées par rapport à un élément complet et type de fracture.

Figure 3 - a) Length (in $\mathrm{mm}$ ) of the faunal remains uncovered at Les Abeilles and b) description of the marrow-bearing elements: length and shaft circumference relative to a complete element and type of fracture.

(3) Confrontation des référentiels disponible dans Soulier 2013. 


\begin{tabular}{|c|c|c|c|c|c|c|}
\hline & \multicolumn{2}{|c|}{ Protoaurignacien } & \multicolumn{2}{|c|}{ Aurignacien ancien } & \multirow{2}{*}{$\mathrm{Chi}^{2}$} & \multirow{2}{*}{$\mathrm{p}$} \\
\hline & NR/NRt & $\% \mathrm{NRt}$ & NR/NRt & $\% \mathrm{NRt}$ & & \\
\hline Fissures longitudinales & $120 / 2561$ & 4,7 & $510 / 12281$ & 4,2 & 1,481 & $>0,05$ \\
\hline Délitement & $32 / 2561$ & 1,2 & $63 / 12281$ & 0,5 & 18,076 & $<0,001$ \\
\hline Dissolution & $127 / 2561$ & 5,0 & 153/12281 & 1,2 & 157,851 & $<0,001$ \\
\hline Lustré & $7 / 2561$ & 0,3 & $6 / 12281$ & 0,0 & 12,202 & $<0,001$ \\
\hline Piétinement & $33 / 2561$ & 1,3 & $3 / 12281$ & 0,0 & 139,952 & $<0,001$ \\
\hline Desquamation & $84 / 2561$ & 3,3 & $187 / 12281$ & 1,5 & 36,506 & $<0,001$ \\
\hline Abrasion & $444 / 2561$ & 17,3 & $172 / 12281$ & 1,4 & 1352,87 & $<0,001$ \\
\hline Corrosion & $29 / 2561$ & 1,1 & 15/12281 & 0,1 & 73,167 & $<0,001$ \\
\hline Concrétion & $17 / 2561$ & 0,7 & 228/12281 & 1,9 & 18,569 & $<0,001$ \\
\hline Vermiculations & $26 / 2561$ & 1,0 & $588 / 12281$ & 4,8 & 76,053 & $<0,001$ \\
\hline Traces de dents & $26 / 2561$ & 1,0 & $21 / 12281$ & 0,2 & 47,846 & $<0,001$ \\
\hline Os brûlés & $136 / 2561$ & 5,3 & $2922 / 12281$ & 23,8 & 442,509 & $<0,001$ \\
\hline Indices de fracturation* & $158 / 803$ & 19,7 & $536 / 1650$ & 32,5 & 43,678 & $<0,001$ \\
\hline Stries* & $233 / 1033$ & 22,6 & $857 / 2687$ & 31,9 & 31,412 & $<0,001$ \\
\hline
\end{tabular}

Tableau 1 - Altérations observées sur les restes de faune des ensembles attribués au Protoaurignacien et à l'Aurignacien ancien des Abeilles en NR, pourcentages et résultats du test du Chi2. * Pourcentages d'indices de fracturation calculés à partir des os à cavité médullaire et pourcentages de stries calculés à partir des restes avec surface observable.

Table 1 - Number of Specimens, percentages and Chi2 test results for bone surface alterations in the Protoaurignacian and Early Aurignacian layers at Les Abeilles. *Percentages of fracture were calculated on marrow-bearing bones. Cutmarks percentages were calculated only using remains with an observable surface.

également davantage marqué par les cupules de dissolution et les traces de piétinement. Les stries de découpe, les encoches de percussion et les os brûlés sont fréquents dans les deux ensembles, et notamment dans l'Aurignacien ancien. En revanche, les pièces portant des traces de manducation sont rares dans les deux ensembles ( $1 \%$ ou moins) ; $28 \%$ des pièces rognées provenant de l'ensemble attribué au Protoaurignacien et $33 \%$ de celles de l'Aurignacien ancien présentent également des stries de découpe.

\section{2 - Spectre faunique}

Les deux ensembles offrent des cortèges fauniques riches (tabl. 2). Les ongulés les plus fréquents dans l'ensemble attribué au Protoaurignacien correspondent aux Bovinés, au Cheval et, dans une moindre mesure, au Renne. L'importance des grands ongulés est accrue lorsque l'on prend en compte les restes seulement attribués à une classe de taille. Les carnivores sont fréquents, et notamment l'Ours et le Renard. Dans l'ensemble attribué à l'Aurignacien ancien, le Renne est l'espèce la plus fréquente, devant les Bovinés et le Cheval ; le Renard est le carnivore le mieux représenté, loin devant l'Ours et l'Hyène.

\section{3 - Âge, sexe et saisons d'abattage (fig. 4)}

Les restes dentaires récoltés dans l'ensemble attribué au Protoaurignacien témoignent de la présence de toutes les classes d'âge pour le Renne, le Cheval et les Bovinés. Sur la base des canines (Barone 1986), deux chevaux mâles seraient présents dans l'ensemble attribué au Protoaurignacien. Des os de fœtus signalent la présence

\begin{tabular}{|l|ccc|ccc|}
\multicolumn{2}{c}{} & \multicolumn{2}{c}{ Protoaurignacien } & \multicolumn{3}{c}{ Aurignacien ancien } \\
\hline Equus caballus & 93 & 18,1 & $3 / 5$ & 322 & 15,9 & $7 / 8$ \\
Bovinae & 95 & 18,5 & $3 / 6$ & 411 & 20,3 & $6 / 10$ \\
Rangifer tarandus & 81 & 15,8 & $3 / 4$ & 872 & 43,1 & $8 / 6$ \\
Cervus elaphus & 21 & 4,1 & $2 / 2$ & 27 & 1,3 & $2 / 2$ \\
Capra sp. & - & - & & 8 & 0,4 & $1 / 2$ \\
Sus scrofa & 5 & 1 & $0 / 2$ & 2 & 0,1 & $0 / 2$ \\
Rupicapra rupicapra & 6 & 1,2 & $1 / 0$ & 17 & 0,8 & $2 / 2$ \\
Equus hydruntinus & 2 & 0,4 & $1 / 1$ & 2 & 0,1 & $0 / 1$ \\
Megaloceros giganteus & 1 & 0,2 & $1 / 0$ & 4 & 0,2 & $1 / 0$ \\
Mammuthus primigenius & 3 & 0,6 & $0 / 1$ & 10 & 0,5 & $0 / 1$ \\
Vulpinae & 85 & 16,6 & $5 / 7$ & 223 & 11 & $5 / 7$ \\
Ursidae & 94 & 18,3 & $1 / 9$ & 70 & 3,5 & $1 / 8$ \\
Crocuta crocuta spelaea & 17 & 3,3 & $1 / 4$ & 29 & 1,4 & $1 / 7$ \\
Felis silvestris & 5 & 1 & $1 / 1$ & 5 & 0,2 & $1 / 2$ \\
Canis lupus & 5 & 1 & $1 / 1$ & 8 & 0,4 & $1 / 1$ \\
Lynx sp. & - & - & & 1 & 0,05 & $1 / 0$ \\
Mustélidés & - & - & & 3 & 0,1 & $1 / 1$ \\
Lagomorphes & - & - & & 7 & 0,3 & $2 / 1$ \\
\hline Total dét. & 513 & & $\mathbf{2 3 / 4 3}$ & $\mathbf{2 0 2 1}$ & & $\mathbf{4 0 / 6 1}$ \\
\hline Ongulés 1/2 & - & & & 53 & & \\
Ongulés 2 & 126 & & & 155 & & \\
Ongulés 2/3 & 179 & & & 90 & & \\
Ongulés 3 & 7 & & & 731 & & \\
Ongulés 3/4 & 315 & & & 8 & & \\
Ongulés 4 & 132 & & & 2 & & \\
Ongulés 4/5 & 1 & & & 1 & & \\
Ongulés 5 & - & & & & & \\
Grands carnivores ind. & 9 & & & & & \\
Moyens carnivores ind. & - & & & & & \\
Carnivores ind. & 2 & & & & & \\
Mammifères ind. & 1277 & & & & & \\
\hline Total & $\mathbf{2 5 6 1}$ & & & & & \\
\hline
\end{tabular}

Tableau 2 - Spectres fauniques des Abeilles en Nombre de Restes (NR), \% par rapport au Nombre de Restes déterminés (\% NRd) et Nombre Minimum d'Individus (NMI). Abréviation : « ind. » = indéterminé.

Table 2 - The faunal spectra at Les Abeilles: Number of Specimens (NR), \% of Number of Identified Specimens (\%NRd) and Minimum Number of Individuals (NMI). Abbreviation: "ind." $=$ indeterminate.

de quatre juments ; les dimensions de ces os indiquent qu'elles auraient été abattues entre début octobre et midécembre. Un os de fœtus de Renne signale la présence d'une femelle dont la saison de mort se situerait entre janvier et mars. L'usure d'une dent déciduale témoigne de la mort d'un jeune renne entre les mois d'août et novembre.

Les profils de mortalité du niveau attribué à l'Aurignacien ancien attestent également de toutes les classes d'âge. Le profil des Bovinés témoigne d'une surreprésentation des individus dans la force de l'âge par rapport aux autres classes. Les dimensions d'un os de fœtus signalent l'abattage d'une femelle boviné gravide en décembrejanvier. Un poulain aurait été abattu entre octobre et mars. Une jument aurait été capturée en novembre et une autre entre janvier et mars.

\section{4 - Représentation squelettique}

Quel que soit le taxon ou le techno-complexe, les os longs et la mandibule sont les éléments les plus fréquents dans les ensembles fauniques (fig. 5a-b). Le crâne est présent 




Figure 4 - a) Profils de mortalité et b) indices de saisonnalité des rennes, chevaux et bovinés aux Abeilles.

Figure 4 - a) Mortality profiles and b) seasonal data for Reindeer, Horse and Bovines at Les Abeilles.

dans des proportions variables selon les taxons et apparaît plus fréquent dans l'ensemble attribué au Protoaurignacien. Pour les chevaux et bovinés des deux ensembles, les métapodes sont moins fréquents que la partie supérieure des membres, tandis qu'ils sont présents dans des proportions élevées dans les profils squelettiques du Renne. Les os des ceintures sont également moins bien représentés pour les grands ongulés que pour le Renne. Un net déficit en squelette axial et en phalanges est visible pour toutes les espèces des deux ensembles. La patella, les carpiens et les tarsiens sont systématiquement sousreprésentés malgré la présence des os adjacents.

La confrontation des différentes portions squelettiques présentes aux Abeilles avec les indices de densité donne de très faibles corrélations, non significatives (fig. $5 \mathrm{c}$ ) ; les profils squelettiques n'apparaissent également pas corrélés avec la masse de viande portée par les ossements (fig. 5c). En revanche, de très fortes corrélations sont observées avec les indices de moelle (quantité de moelle contenue dans la cavité médullaire et quantité totale de graisses insaturées : fig. $5 \mathrm{c}$ ). Les profils squelettiques ne semblent donc pas illustrer la présence de carcasses complètes au sein de la grotte, mais de segments de carcasses.

\section{5 - Traces de boucherie}

\subsection{1 - Les stries de découpe (fig. 6)}

Les stries de découpe sont fréquentes dans les deux ensembles, et notamment dans le niveau attribué à l'Aurignacien ancien (cf. tabl. 1).
Peu de stries relevant de l'étape d'éviscération ont été relevées dans les deux ensembles. Elles ont été observées uniquement sur des côtes de grands ongulés (Cheval ou Bovinés) dans le niveau attribué au Protoaurignacien et sur le Renne et les Bovinés dans l'Aurignacien ancien.

Les stries de dépouillement sont relativement fréquentes dans les deux ensembles.

Dans l'ensemble attribué au Protoaurignacien, des stries orientées mésio-distalement sur la face vestibulaire d'une dent jugale de Renne indiquent une incision de la peau au niveau de la gueule. Pour cette même espèce, une strie longitudinale localisée en face postérieure d'une première phalange témoigne d'une incision de la peau au niveau de l'acropode. Pour le Cheval, des stries localisées sur les dents indiquent le retrait de la peau d'au moins une mandibule et de celle du crâne d'au moins un jeune et un adulte. Deux longues stries localisées en partie distale de la diaphyse d'un métatarsien pourraient avoir été créées lors du retrait de la peau. Les restes dentaires et un fragment de mandibule de Bovinés témoignent que la peau de la mandibule a été retirée sur au moins trois individus. Une deuxième phalange de Bovinés porte des stries transverses en face abaxiale. L'ensemble protoaurignacien fournit également des indices de dépouillement de carnivores : la peau du crâne d'au moins deux ours, un loup, un chat et une hyène a été retirée. Une partie distale de fibula d'Ours porte une série de stries obliques qui pourraient avoir été créées lors du retrait de la peau (cf. Mallye 2011).

Dans l'ensemble attribué à l'Aurignacien ancien, les traces relatives au dépouillement sont nombreuses sur les restes de Renne et ont été observées sur la plupart des éléments pouvant potentiellement être affectés par ce type 


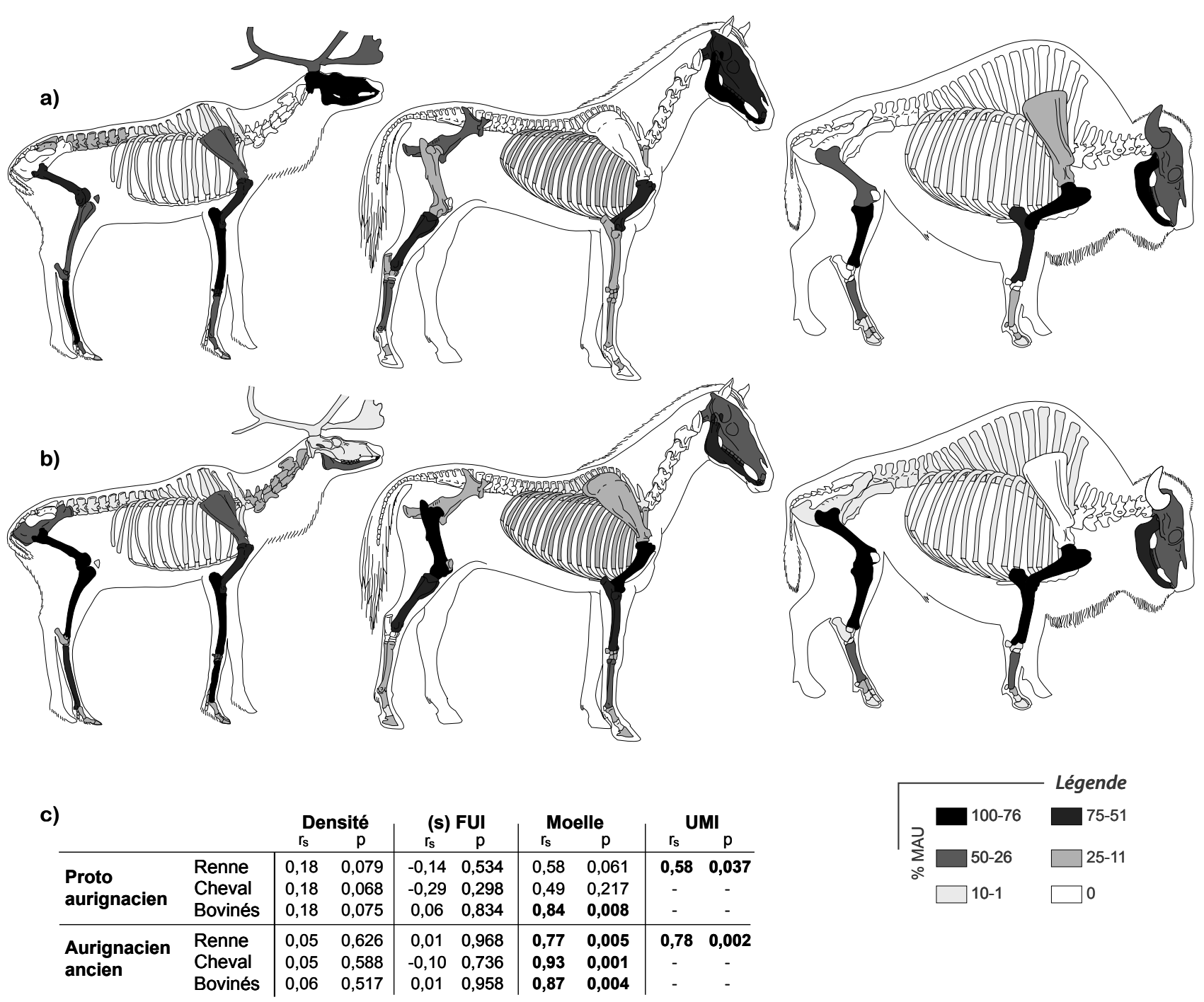

Figure 5 - Profils squelettiques en \% MAU du a) Protoaurignacien et b) de l'Aurignacien ancien aux Abeilles. c) corrélations de Spearman sur la fréquence des os en fonction de leur densité, du (s) FUI (Food Utility Index standardisé), de la quantité de moelle osseuse contenue dans les os à cavité médullaire et de l'UMI (quantité totale de graisses insaturées). En gras : résultats significatifs au seuil de 0,05 .

Figure 5 - Skeletal profiles in \%MAU for the a) Protoaurignacian and b) Early Aurignacian layers at Les Abeilles. c) Spearman correlations on element frequencies compared to their density value, the (S)FUI (Standardized Food Utility Index), marrow cavity volume, and the quantity of unsaturated fatty acids (UMI, Unsaturated Marrow Index). Statistically significant results (at the 0.05 level) are shown in bold.

d'opération. Si le retrait de la peau du crâne et de la mandibule est documenté, la majorité des indices de dépouillement ont été observés sur les os du métapode. Au niveau du métacarpien, deux zones d'entame sont documentées via des stries transverses situées en parties proximale et médiale de la diaphyse. Des stries obliques témoignent de gestes obliques pratiqués pour décoller la peau. Sur le métatarsien, des stries transverses se poursuivant sur les faces médiale, postérieure et latérale signalent une zone d'entame circulaire en partie distale de la diaphyse, tandis que des stries longitudinales en face médiale illustrent un geste remontant depuis la zone d'entame vers l'incision ventrale d'éviscération. Des zones d'entame circulaire sont également documentées sur les premières et deuxièmes phalanges de cette espèce. Pour le Cheval, des stries de dépouillement sont présentes sur le crâne d'un poulain et sur la mandibule d'au moins trois individus dont un jeune. Au niveau des pattes, une zone d'incision circulaire est documentée en milieu de diaphyse d'un métacarpien. Des stries de dépouillement ont également été observées sur la mandibule d'au moins deux bovinés. Sur les os du métapode, des entames circulaires apparaissent en partie proximale et des stries obliques liées au décollement de la peau sont présentes sur toute la hauteur de la diaphyse. Des stries transverses sur la diaphyse du tibia, en partie distale, pourraient traduire une 


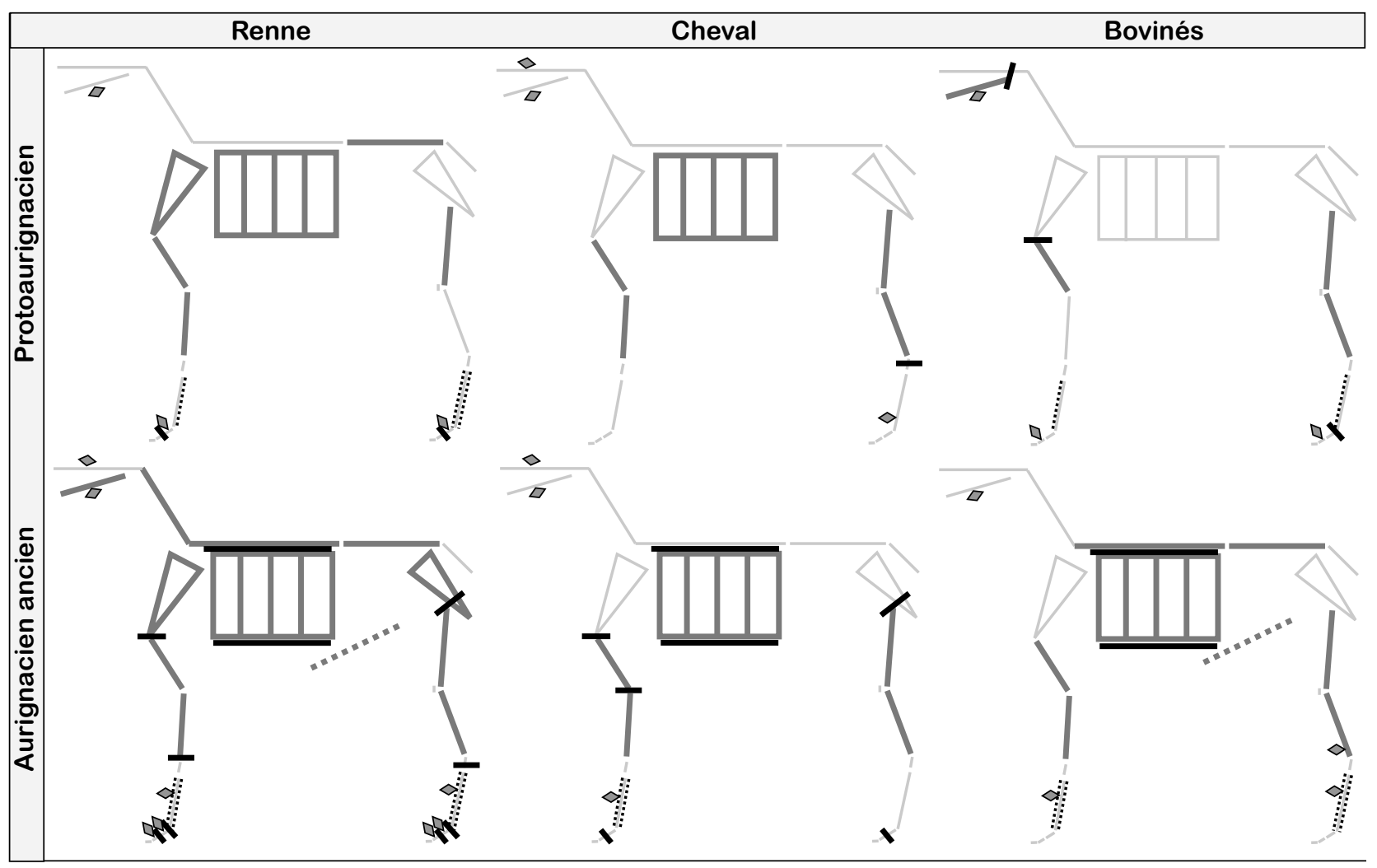

Légende - décharnement $\diamond$ dépouillement " - éviscération - désarticulation ... prélèv. tendons

Figure 6 - Synthèse des différents types de stries observées sur les restes de rennes, chevaux et bovinés des Abeilles.

Figure 6 - Summary of the different types of cutmarks observed on Reindeer, Horse and Bovines at Les Abeilles.

zone d'entame située à la base des éléments charnus. Pour le Renard, les stries de dépouillement témoignent du retrait de la peau du crâne d'au moins un renardeau et d'un sujet adulte, et de la mandibule. Des stries transverses localisées sur plusieurs premières et deuxièmes phalanges, ainsi que sur des os du métapode, signalent une incision réalisée en bas-de-pattes pour au moins deux renards. Enfin, un fragment de maxillaire atteste du dépouillement d'un crâne de Loup.

Les stries de décharnement, courtes et quasi exclusivement transverses ou obliques, sont fréquentes dans les deux ensembles.

Pour les trois ongulés principaux de l'ensemble attribué au Protoaurignacien, elles sont présentes sur tous les os longs charnus, à l'exception du tibia de Renne et du radius de Bovinés. Elles ont également été identifiées sur un humérus de Cerf et un tibia de Renard. Le décharnement des côtes est attesté pour le Renne, le Cheval et le Renard. Au niveau des ceintures, seule la scapula de Renne porte des stries diagnostiques du décharnement. Le prélèvement de la langue est uniquement avéré pour les Bovinés, grâce à la présence de stries localisées sur la face linguale de dents jugales.

Dans l'ensemble attribué à l'Aurignacien ancien, des stries de décharnement sont présentes sur les côtes de Renne,
Cheval, Bovinés et Renard. Plusieurs fragments de vertèbres thoraciques et/ou lombaires de Renne, Bovinés et Bouquetin portent des stries typiquement produites lors du prélèvement de la longe. Le décharnement du cou et des ceintures est en revanche uniquement attesté pour le Renne. Tous les os longs charnus de Renne, Cheval et Bovinés portent des stries de décharnement. Les os des pattes d'au moins trois renards présentent également ce type de stries. Pour les autres espèces, les stries de décharnement sont plus rares ; elles ont néanmoins été observées sur l'humérus, le radius et le tibia d'un cerf, le radius d'un mégacéros et d'un isard ainsi que sur le fémur et le tibia d'un lièvre. Enfin, des stries en face linguale d'un fragment de mandibule de Renne signalent le prélèvement de la langue.

Les extrémités articulaires sont peu fréquentes dans les deux ensembles ce qui limite fortement les possibilités d'observer des stries de désarticulation. Relativement au NRt de chacun des deux ensembles, les extrémités articulaires - qui correspondent aux portions les plus susceptibles de présenter ce type de stries - sont légèrement plus fréquentes dans l'ensemble attribué au Protoaurignacien $(4,41 \%$ du NRt) que dans l'Aurignacien ancien $(3,13 \%$ du NRt). Les stries de désarticulation sont 
néanmoins plus abondantes dans le niveau attribué à l'Aurignacien ancien.

Dans l'ensemble attribué au Protoaurignacien, seuls les restes de Bovinés attestent d'une désarticulation entre la mandibule et le crâne. Des stries de désarticulation sont en revanche attestées au niveau des os longs de Bovinés et de Chevaux et à hauteur des phalanges pour le Renne. Le squelette axial postcrânien des ongulés ne présente aucune strie de désarticulation. Une profonde incision sur la partie crâniale d'une vertèbre caudale de Renard signale que, pour cette espèce, une désarticulation entre le squelette axial et la queue a été effectuée.

Dans l'ensemble attribué à l'Aurignacien ancien, aucune trace de désarticulation n'a été observée au niveau de la tête. Plusieurs stries, localisées au niveau des côtes et des vertèbres, documentent la séparation de la colonne vertébrale et des côtes. Des stries profondes situées en partie distale des côtes indiquent que celles-ci ont été désolidarisées du sternum pour le Cheval, le Renne et les Bovinés. Au niveau des membres, aucun reste de Bovinés ne porte de stries relatives à la désarticulation tandis que cette opération est avérée pour le Renne et le Cheval. Des stries de désarticulation entre le pelvis et le fémur ont été observées sur une tête fémorale de Renard. De profondes stries localisées sur des calcanéums indiquent une désarticulation pratiquée au niveau du bloc tarsien pour le Lièvre et le Bouquetin.

Le sectionnement des tendons est attesté dans les deux ensembles via des stries courtes et transverses localisées au niveau des gouttières des métapodes. Dans l'ensemble Protoaurignacien, ces stries sont présentes sur les métacarpiens et les métatarsiens de Renne, de Bovinés et de Cerf. Pour l'ensemble Aurignacien ancien, le sectionnement des tendons fléchisseurs et extenseurs a été pratiqué sur le Renne, les Bovinés, le Cheval et le Cerf.

\subsection{2 - Les indices de fracturation des os}

Tous les os longs d'ongulés des deux ensembles des Abeilles sont fragmentés ; les portions articulaires d'os longs sont rares et aucune n'a été récoltée entière. L'analyse des bords de fracture indique que la fragmentation des os à cavité médullaire s'est essentiellement produite sur os frais pour les deux ensembles (cf. fig. 3b).

Dans l'ensemble attribué au Protoaurignacien, les seuls os d'ongulés à cavité médullaire complets correspondent à quatre phalanges ( 2 premières et 2 deuxièmes). Près de 20 $\%$ des os à cavité médullaire portent des encoches de percussion ; ces traces ont été observées sur le Renne, le Cheval, les Bovinés, le Cerf et potentiellement sur le Renard. Certaines encoches de percussion, localisées à proximité des extrémités articulaires d'os longs, pourraient traduire une volonté d'isoler les extrémités articulaires. Si les os longs sont les éléments les plus affectés par les encoches, elles ont aussi été observées sur deux premières phalanges de Renne et sur un fragment de mandibule de boviné. Cinq os courts d'ongulés sur douze sont complets dans cet ensemble. Plusieurs éléments spongieux non déterminés et de petites dimensions - pour la plupart non brûlés - ont été récoltés $(<25 \mathrm{~mm}=6 \%$ du NRt).

Dans le niveau attribué à l'Aurignacien ancien, hormis dix phalanges $(\mathrm{Ph} 1 \mathrm{co}=4 ; \mathrm{PH} 2 \mathrm{Co}=6)$, tous les os à cavité médullaire d'ongulés sont fragmentés. Les encoches de percussion sont présentes sur $32,5 \%$ des os à cavité médullaire et ont été observées sur les restes de Renne, Cheval, Bovinés, Cerf, Isard et, avec un plus grand degré d'incertitude, sur le Renard. Elles sont essentiellement localisées sur les diaphyses d'os longs mais ont aussi été observées sur quatre fragments de mandibule de Renne et quatre de Bovinés. Aucune encoche n'a été observée sur les fragments de mandibule de Cheval mais douze dents jugales inférieures $(\mathrm{NMI}=5$ ) ont un fût fendu oblique qui pourrait avoir été brisé lors de la fracturation de la mandibule (e.g. Morel et Müller 1997 ; Costamagno 1999 ; Outram 2005) ; plusieurs dents jugales supérieures présentent également des fûts fendus obliques pouvant indiquer l'ouverture d'un crâne de cheval. Quatre premières phalanges et cinq deuxièmes phalanges (Renne et Bovinés) présentent des encoches de percussion. Neuf fragments osseux portent des impacts et des contreimpacts, documentant de fait l'utilisation d'une enclume. Des alignements d'encoches de percussion ont été observés sur douze fragments diaphysaires (fig. 7). Ces pièces portent quasi systématiquement une plage de retouchoir ; ces alignements d'encoches pourraient ainsi attester d'un débitage visant à contrôler la morphologie du support. Un impact de percussion a été identifié sur une tête humérale de Renne et plusieurs encoches de percussion sont situées à proximité immédiate d'extrémités articulaires d'os longs. Trois os des blocs carpiens et tarsiens présentent des bords de fracture très rectilignes et lisses suggérant le caractère intentionnel de leur fracturation (cf. Darwent et Lyman 2002) ; cette fracturation n'a toutefois pas été systématique puisque la moitié des os courts ont été récoltés complets (13 sur 24). Les éléments spongieux indéterminés de moins de $25 \mathrm{~mm}$ constituent $26 \%$ du NRt. Dans cet échantillon, $60 \%$ des pièces ne présentent aucune trace de chauffe.

\section{6 - Les os brûlés}

La présence d'os brûlés a été relevée dans les deux ensembles ; ce type de matériel est plus abondant dans le niveau attribué à l'Aurignacien ancien (23,8 \% du NRt) que dans le Protoaurignacien (5,3\% du NRt).

La grande majorité des pièces brûlées récoltées dans l'ensemble attribué au Protoaurignacien sont au moins carbonisées (tabl. 3) et de petites dimensions puisque $93,4 \%$ d'entre-elles mesurent moins de $2 \mathrm{~cm}$ de long. Ce matériel brûlé est composé à $69 \%$ de structure compacte, $28 \%$ d'éléments spongieux, $2 \%$ de restes dentaires et $1 \%$ de squelette axial. Le taux d'os brûlés à structure spongieuse semble trop faible pour pouvoir interpréter leur présence comme résultant d'une utilisation de la matière osseuse comme combustible. L'absence d'informations précises sur l'utilisation d'un tamisage ne permet 


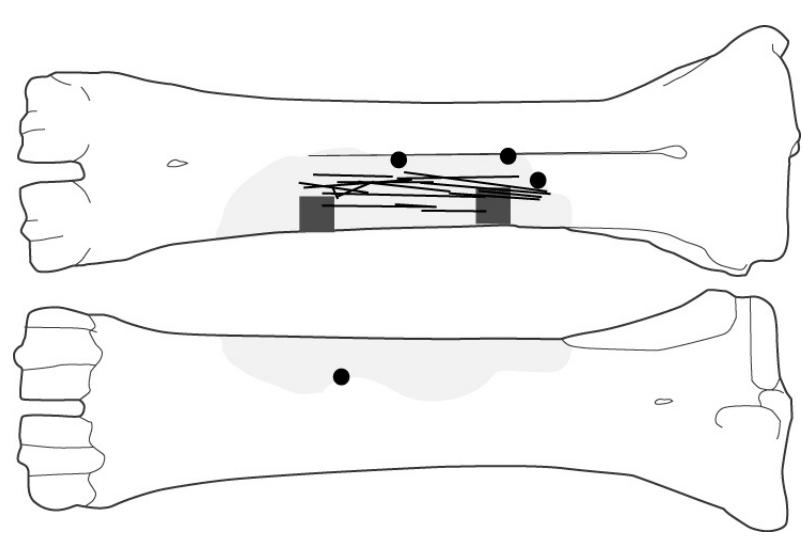

Figure 7 - Exemple d'une pièce avec alignement d'encoches de percussion (points noirs). En gris clair : fragment, carré gris foncé : plage de retouchoir, trait noir épais : strie de raclage.

Figure 7 - Example of a bone fragment with aligned percussion notches (black dots). In light grey: the fragment; dark grey square: area used as retoucher, thick black line: scrape marks.

néanmoins pas de savoir si les petits fragments d'os spongieux carbonisés ont été ramassés de manière exhaustive. Dans cet ensemble, un fragment de côte d'ongulé de grande taille présente une brûlure distale et atteste qu'un morceau de cage thoracique a été préparé grillé. Des traces de brûlure légères ont été observées sur la surface occlusale de trois dents de Renne. De par leur localisation, elles pourraient indiquer qu'une tête, dont la mandibule aurait été préalablement désarticulée, a été directement déposée dans un foyer, potentiellement pour cuire la cervelle.

Dans l'ensemble attribué à l'Aurignacien ancien, la quasi totalité des pièces correspondent à du tissu compact ou spongieux (respectivement $51 \%$ et $46 \%$ des pièces brûlées) ; à ces pièces s'ajoutent des fragments de squelette axial, de crâne et des restes dentaires en quantité négligeable. Ce matériel brûlé est très fragmenté $(92,8 \%$ des pièces mesurent moins de $2 \mathrm{~cm}$ ) et correspond essentiellement à des os au moins carbonisés (tabl. 3). Ces données concordent avec une exploitation de l'os comme matière combustible. Les pièces brûlées ayant pu être déterminées appartiennent essentiellement au Renne et à des éléments riches en spongiosa (extrémités articulaires, os courts, ceintures : cf. Soulier 2013). L'effort de détermination de ces pièces brûlées a notamment permis de souligner que les os des blocs carpiens et tarsiens de Renne sont «fréquemment 》 brûlés $(28,6 \% \mathrm{NRd}$ de carpiens et 33,3\% du NRd de tarsiens) ce qui pourrait offrir une explication quant à la sous-représentation de ces éléments dans le profil squelettique (cf. fig. 5). Enfin, une

\begin{tabular}{|c|c|c|c|c|}
\hline & \multicolumn{2}{|c|}{ Protoaurignacien } & \multicolumn{2}{|c|}{ Aurignacien ancien } \\
\hline & NR & \% d'os brûlés & NR & \% d'os brûlés \\
\hline 1 & 12 & 8,8 & 12 & 0,4 \\
\hline$\because 2$ & 112 & 82,4 & 2778 & 95,1 \\
\hline 常 3 & 12 & 8,8 & 27 & 0,9 \\
\hline 4 & 0 & 0 & 105 & 3,6 \\
\hline
\end{tabular}

Tableau 3 - Caractérisation des os brûlés récoltés aux Abeilles dans les ensembles attribués au Protoaurignacien et à l'Aurignacien ancien en Nombre de Restes et pourcentage d'os brûlés. D'après les stades de Costamagno et al. 2009.

Table 3 - Characterization of the burned remains uncovered in the Protoaurignacian and Early Aurignacian layers at Les Abeilles with values calculated as a function of the Number of Specimens and the percentage of burned bones. Combustion stages are as in Costamagno et al. 2009.

tête de côte de Cheval et un éclat diaphysaire d'os long d'ongulé de taille moyenne portent des brûlures à leurs extrémités attestant de la consommation de viande grillée non désossée.

\section{7 - L'industrie en matière dure animale}

La présence d'industrie en matière dure animale est attestée dans les deux ensembles. Par rapport au premier inventaire sur l'industrie en matière dure et la parure des Abeilles (Barandiarán 2006), la reprise d'étude de la faune a permis d'isoler de nombreuses pièces supplémentaires qui viennent grossir considérablement le corpus ${ }^{4}$.

L'industrie sur faune récoltée dans le niveau attribué au Protoaurignacien, originellement composée de seulement six pièces (op. cit.), s'est enrichie de treize retouchoirs et d'un outil intermédiaire (fig. 8a). En revanche, les lissoirs décrits auparavant semblent n'être que des pièces très émoussées, de même que la pendeloque, pièce sur laquelle la perforation a été confondue avec une cupule de dissolution. Les espèces utilisées comme support pour confectionner l'industrie correspondent aux Bovinés, au Cheval, au Renne, au Cerf et au Mammouth, auxquelles s'ajoutent quelques pièces de grands ongulés et de cervidés indéterminés. La majorité de cette industrie est réalisée sur os et correspond à des retouchoirs. Un outil intermédiaire en os d'ongulé de grande taille, une pointe de sagaie sur bois de cervidé et deux bâtonnets en ivoire complètent cette série. La longueur des éclats sélectionnés comme supports de retouchoirs varie entre 35 et $125 \mathrm{~mm}$ (fig. 8b) ; cinq d'entre eux portent un aménagement de la surface par raclage du périoste. En raison du faible nombre de restes, aucune zone éventuellement exploitée préférentiellement pour la plage de retouche ne peut être perçue; nous pouvons simplement souligner que le milieu de diaphyse postérieure de l'humérus a été exploité à la fois chez le Cerf et les Bovinés.

(4) Une reprise d'étude de ce matériel est actuellement en cours par É. Tartar. 
a)

\begin{tabular}{llcccccc} 
& & Renne & Cheval & Bovinés & Cerf & Mammouth & cervidé ind. ong. $3 / 4$ \\
\hline \multirow{4}{*}{ retouchoir } & $\begin{array}{l}\text { Côtes } \\
\text { humérus }\end{array}$ & & 1 & & & & 1 \\
& $\begin{array}{l}\text { métacarpien } \\
\text { fémur }\end{array}$ & 1 & & 2 & 2 & & \\
& tibia & & 1 & 1 & & & 1 \\
& os long ind. & & 1 & & & 1 \\
\hline sagaie & bois & & 1 & & & 1 \\
\hline bâtonnet & ivoire & & & & & 1 \\
\hline outil intermédiaire & os & & & & & 1 \\
\end{tabular}

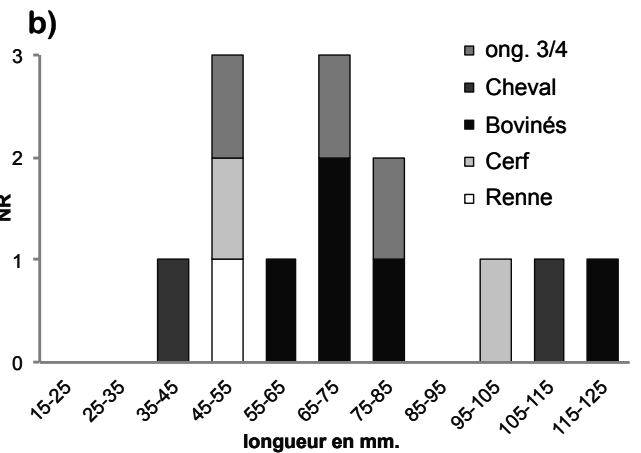

Figure 8 - a) inventaire des restes fauniques travaillés et b) longueur des fragments osseux utilisés comme retouchoirs dans le niveau attribué au Protoaurignacien des Abeilles.

Figure 8 - a) List of the worked faunal remains and b) lengths of the retoucher blanks recovered in the Early Aurignacien layer at les Abeilles.

Pour l'ensemble attribué à l'Aurignacien ancien, le corpus de restes fauniques transformés à des fins utilitaire ou symbolique (fig. 9a) se compose de 148 éléments (plus potentiellement 7 autres) contre seulement 48 décrits auparavant. Cette industrie a été réalisée à partir de matière dure provenant majoritairement de Bovinés (fig. 9a et tabl. 4) et, dans une moindre mesure, de Renne, Cheval, Cerf, Mammouth, Renard et Ours.

Les os longs ont servi de supports pour la confection de plus de $70 \%$ de l'industrie. Des fragments de mandibule de Renne et de Bovinés, des métapodes vestigiels, des côtes de grands ongulés, du bois de cervidé, des dents de carnivores et de l'ivoire de Mammouth ont plus rarement été utilisés pour réaliser des lissoirs, des poinçons, des pointes de sagaies, des éléments de parure, etc. Cette industrie se compose néanmoins majoritairement de retouchoirs, dont les supports mesurent généralement entre 55 et $85 \mathrm{~mm}$ (fig. 9b). Un tiers des retouchoirs présente un aménagement de la surface osseuse par raclage, généralement pratiqué longitudinalement à l'axe de l'os. Quatorze retouchoirs possèdent une double plage et un autre en porte trois. Ces retouchoirs multiples ont surtout été observés sur le fémur (33\% du NRt retouchoirs

\begin{tabular}{ll|ll} 
& & \multicolumn{1}{c}{ Chi $^{\mathbf{2}}$} & \multicolumn{1}{c}{$\mathbf{p}$} \\
\hline Renne & Bovinés & 37,0355 & $1,16 \mathrm{E}-09$ \\
Bovinés & Cheval & 10,1027 & 0,001481 \\
Cheval & Renne & 2,3308 & 0,1268
\end{tabular}

Tableau 4 - Résultats des tests du Chi2 sur le nombre de pièces utilisées comme retouchoir dans l'Aurignacien ancien des Abeilles (Bovinés, Renne, Cheval) par rapport au NR déterminés total par taxon.

Table 4 - Chi2 test results for the number of remains used as retouchers in the Early Aurignacian layer at Les Abeilles (Bovines, Reindeer, Horse). Results were compared to the total NISP for a given species. multiples) et exclusivement sur des os d'ongulés de grande taille : neuf sur Bovinés (dont le retouchoir triple) et cinq sur Cheval.

Une récurrence dans la localisation des plages de retouchoir peut être perçue. Cinq des huit plages de retouchoir observées sur le radio-ulnaire de Bovinés sont situées en milieu de diaphyse antérieure (fig.10a) et, pour le fémur, six des douze plages sont localisées dans le tiers distal de la diaphyse latérale (fig.10b). Ces récurrences sont essentiellement visibles par taxon et pourraient être directement liées aux propriétés spécifiques des os de chaque espèce (degré de concavité/convexité, épaisseur et texture de la diaphyse, etc.). Une récurrence en milieu de diaphyse d'humérus, sur la face latérale, apparaît néanmoins chez les Bovinés, le Cheval et le Renne (fig.10c). La présence d'encoches de percussion alignées sur sept retouchoirs pourrait suggérer un débitage contrôlé de certains supports.

\section{5 - Discussion}

Les nouvelles données archéozoologiques obtenues pour les ensembles attribués au Protoaurignacien et à l'Aurignacien ancien des Abeilles offrent des éléments de discussion quant aux modalités d'exploitation de la faune au Paléolithique supérieur ancien. En sus d'une exploitation alimentaire, une partie de la faune a été exploitée comme support d'industrie et de parure. L'analyse combinée de l'ensemble des restes fauniques permet dès lors de reconstruire les chaînes opératoires d'exploitation du gibier et d'appréhender le degré d'imbrication entre les sphères alimentaire, technique et symbolique.

\section{1 - Spectre faunique et agent d'accumulation}

Bien que globalement similaires, les cortèges fauniques se distinguent néanmoins en termes d'abondance d'espèces. Le spectre faunique est dominé par le trio Bovinés-ChevalRenne au Protoaurignacien tandis qu'à l'Aurignacien ancien l'importance du Renne augmente au détriment des 
a)

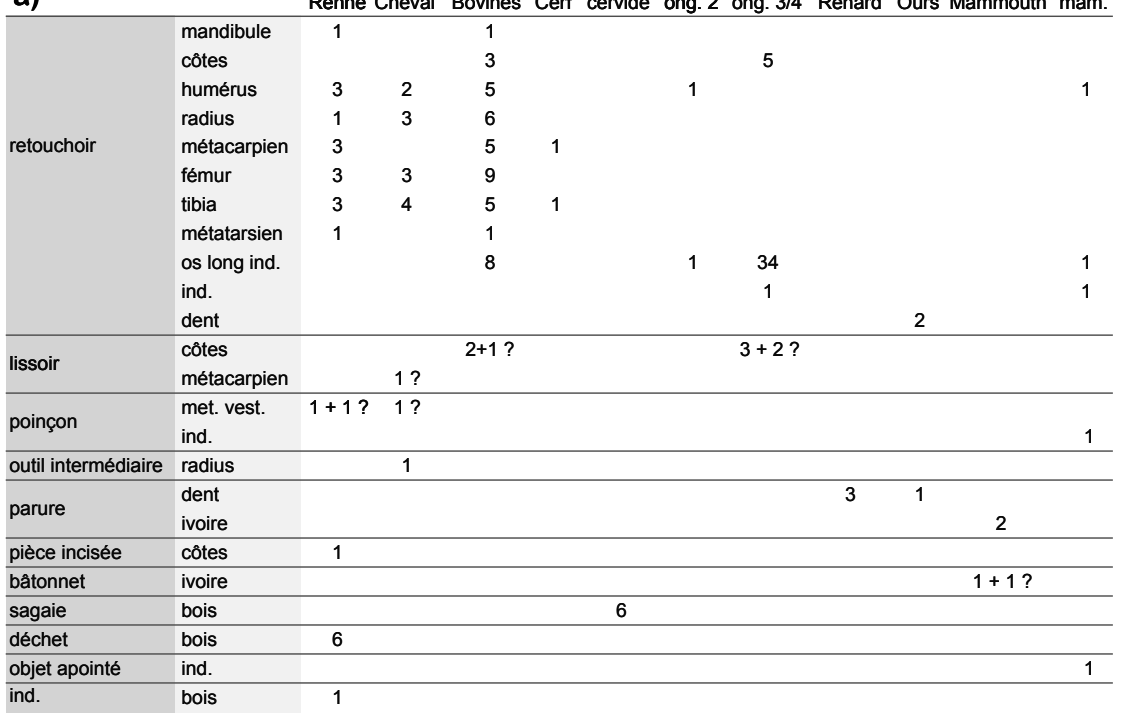

b)



Figure 9 - a) inventaire des restes fauniques travaillés et b) longueur des fragments osseux utilisés comme retouchoirs dans le niveau attribué à l'Aurignacien ancien des Abeilles.

Figure 9 - a) List of the worked faunal remains and b) lengths of the retoucher blanks recovered in the Early Aurignacien layer at les Abeilles.
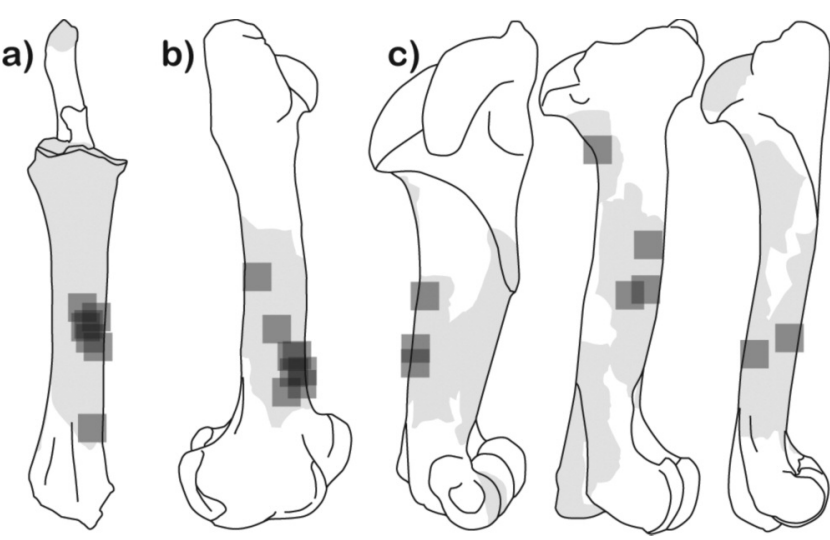

Figure 10 - Récurrence des zones exploitées comme retouchoir dans le niveau attribué à l'Aurignacien ancien des Abeilles sur a) le radio-ulnaire de Bovinés, b) le fémur de Bovinés et c) l'humérus de Bovinés, Cheval et Renne.

Figure 10 - Patterns in retoucher surface areas in the Les Abeilles Early Aurignacian assemblage on a) Bovine radio-ulna, b) Bovine femur and c) Bovine, Horse and Reindeer humerus.

chevaux et des bovinés. Cette augmentation du Renne pourrait davantage être conditionnée par une plus forte présence de cette espèce dans l'environnement (Grayson, Delpech, Rigaud 2001 ; Grayson et Delpech 2002, 2006 ; Morin 2008, 2012 ; Discamps 2011 ; Discamps et al. 2011) que d'un attrait plus prononcé pour cette espèce de la part des chasseurs de l'Aurignacien ancien des Abeilles.

Si l'Ours et le Renard tiennent une place importante dans les spectres fauniques, ces deux espèces ne semblent pas être à l'origine des stocks osseux de par leur régime alimentaire (cf. Bocherens, Fizet, Mariotti 1990 ; Larivière et Pasitschniak-Arts 1996). L'Hyène est peu présente dans les deux ensembles et les traces de morsures sont rares et nettement en deçà des taux issus des repaires d'hyènes, $y$ compris ceux fouillés récemment (e.g. Villa et al. 2004 ; Beauval et Morin 2010 ; Discamps 2011). Plusieurs des pièces rognées portent par ailleurs des stries de découpe, arguant en faveur de stocks osseux accumulés par l'Homme, sur lesquels les carnivores seraient intervenus très marginalement.

\section{2 - L'exploitation alimentaire du gibier}

Une exploitation alimentaire est documentée dans les deux ensembles pour le Renne, le Cheval, les Bovinés, le Cerf et le Renard. Le cortège d'espèces exploitées à des fins alimentaires est plus important dans l'ensemble attribué à l'Aurignacien ancien, puisqu'à ces espèces s'ajoutent le Bouquetin, l'Isard, le Mégacéros et le Lièvre. Les stries étant des épiphénomènes, on ne peut exclure que l'absence de traces d'exploitation alimentaire ne soit pas simplement liée à la taille des échantillons puisque le NR de ces espèces est moins élevé dans l'ensemble attribué au Protoaurignacien que dans l'Aurignacien ancien.

Les rennes, chevaux et bovinés ont été abattus indépendamment de leur âge bien que les très jeunes rennes et bovinés semblent avoir été évités. Les chasseurs paraissent en revanche avoir préférentiellement ciblé les bovinés dans la force de l'âge à l'Aurignacien ancien. Les indices de saisonnalité témoignent d'une récurrence dans la période d'occupation du gisement entre les deux ensembles, à l'automne et en hiver. Ces saisons, par rapport au sexe des proies capturées, attestent de 
l'exploitation de proies intéressantes en termes d'apports en masse carnée et graisseuse. À l'automne, les proies sont en bonne condition sanitaire et, pour les rennes et les bovinés chassés en hiver, les femelles sont en meilleure condition physique que les mâles (Soper 1941 ; Kelsall 1968).

Les profils squelettiques sont relativement homogènes entre les deux ensembles et indiquent que le gibier a essentiellement été transporté jusqu'à la grotte sous forme de quartiers et non de carcasses complètes. Les chasseurs semblent avoir choisi d'emporter préférentiellement les éléments riches en moelle osseuse au détriment du squelette axial, et ce, quel que soit le taxon. Le degré de sélection des éléments emportés semble corrélé à la taille du gibier ; les membres apparaissent relativement complets pour le Renne tandis que pour les grands ongulés, les os des ceintures et des bas-de-pattes sont nettement moins fréquents que les os longs charnus. Relativement à la bonne condition physique des proies abattues, l'emport de ces parties lourdes par rapport à leur apport nutritif limité peut expliquer leur abandon. Par ailleurs, de nombreuses études ethnographiques signalent que le rôtissage de grils costaux et la consommation de la moelle des os du métapode sont fréquents sur les sites d'abattage (e.g. Binford 1978 ; Bunn, Bartram, Kroll 1988 ; Kent 1993). Plusieurs fœtus ont été identifiés dans les deux ensembles ; cinq fœtus sont attestés dans le niveau attribué au Protoaurignacien $(N R=16)$ et trois sont présents dans l'ensemble attribué à l'Aurignacien ancien $(N R=14)$. Ces fœtus étaient à un stade de développement relativement avancé et correspondent surtout à des grands ongulés. Au regard des modes de transport adoptés (cf. supra), ces fœtus ont été délibérément amenés dans la grotte par les chasseurs.

Toutes les étapes-clés du processus de boucherie sont documentées dans les deux ensembles : éviscération, retrait de la peau, décharnement, désarticulation, extraction des tendons et prélèvement de la moelle.

Le décharnement correspond à l'activité qui a laissé le plus de traces sur le matériel osseux. Dans les deux ensembles, les muscles ont été prélevés sur la plupart des éléments charnus et la langue a été récupérée. Le décharnement a été pratiqué sur le Renne, le Cheval, les Bovinés, le Cerf et le Renard dans les deux ensembles, auxquels s'ajoutent le Cerf, le Bouquetin, l'Isard, le Mégacéros et le Lièvre dans le niveau attribué à l'Aurignacien ancien. Dans les deux ensembles, quelques brûlures distales localisées sur des côtes et des os longs indiquent que certains quartiers de viande ont été rôtis sans avoir été préalablement désossés. Aucune strie ne permet d'attester du prélèvement de la viande des fœtus ; cette viande est toutefois très tendre et fortement appréciée de nombreux groupes humains subactuels (e.g. Jenness 1923 ; Speck 1935 ; Burch 1972 ; Hungry Wolf 1980 ; Ingstad 1992 ; Houston S., Ball, Houston M. 2003).

La mise en pièces des carcasses à l'aide d'outils tranchants est davantage documentée dans l'ensemble attribué à l'Aurignacien ancien. On ne peut toutefois exclure l'emploi d'une désarticulation par percussion dans l'ensemble attribué au Protoaurignacien puisque certaines encoches de percussion sont localisées à proximité des extrémités articulaires. Certaines pratiques de boucherie, telles que la fabrication de biltong, ne nécessitent pas - ou peu - de désarticulation (e.g. Nilssen 2000). En revanche, si l'on souhaite récupérer la moelle osseuse contenue dans la cavité médullaire des os, la mise en pièces des membres facilite la fracturation des ossements (e.g. Jin et Mills 2011).

La fracturation des ossements à cavité médullaire pour en extraire la moelle est documentée dans les deux ensembles. Les espèces concernées par cette activité sont le Renne, les Bovinés, le Cheval, le Cerf et éventuellement le Renard dans les deux ensembles, auxquelles s'ajoute I'Isard dans le niveau attribué à l'Aurignacien ancien. Cette opération a été pratiquée sur tous les éléments pourvoyeurs de moelle : os longs, phalanges et mandibule. La récupération de moelle osseuse n'a cependant pas été conduite de manière exhaustive dans les deux ensembles puisque certaines phalanges ont été récupérées complètes. Ces éléments étant les derniers pourvoyeurs de moelle chez les ongulés en cas de sous-nutrition (Speth 1983 ; Morin 2007), cette observation appuie l'idée d'exploitation d'individus en bonne condition physique dans les deux ensembles. Dans l'ensemble attribué à l'Aurignacien ancien, cette fracturation pourrait également avoir été conduite sur le crâne, afin de récupérer la cervelle. Dans les deux ensembles, les grands ongulés, et notamment les Bovinés, sont ceux qui portent le plus d'encoches de percussion. Cette observation peut être mise en relation avec la lisibilité des encoches de percussion, directement liée à l'épaisseur de la corticale des ossements : plus la corticale est épaisse, plus les encoches sont marquées (Marean et Cleghorn 2003). Les encoches de percussion sont beaucoup plus abondantes dans l'ensemble attribué à l'Aurignacien ancien. Cette augmentation du taux d'encoches est nettement visible sur le Cheval, avec un pourcentage deux fois plus élevé que dans le Protoaurignacien. Les os à cavité médullaire complets sont néanmoins tout aussi rares dans les deux ensembles, ce qui ne permet pas de supposer une récupération de la moelle moins importante dans l'ensemble Protoaurignacien. La plus forte présence d'indices de percussion pourrait en revanche relever du mode de fracturation employé par les bouchers. L'utilisation d'une enclume est attestée dans le niveau attribué à l'Aurignacien ancien et l'on peut se demander si cette technique n'engendrerait pas une meilleure visibilité des indices de percussion. Plusieurs éléments pouvant traduire une récupération de la graisse contenue dans les tissus spongieux (e.g. Leechman 1951 ; Lupo et Schmitt 1997 ; Outram 2001, 2002, 2005 ; Church et Lyman 2003 ; SaintGermain 2005) ont été observés, et notamment dans l'ensemble attribué à l'Aurignacien ancien : fragments spongieux de petite taille non brûlés, os courts fendus, percussion sur des extrémités articulaires. La préparation de bouillons gras est néanmoins encore trop délicate à percevoir pour pouvoir affirmer que cette pratique a eu lieu aux Abeilles. 


\section{3 - L'exploitation technique de la faune}

Une large gamme d'espèces a été exploitée comme support d'industrie et de parure. Le Renne, les Bovinés, le Cheval, le Cerf et le Mammouth ont été exploités dans les deux ensembles. À ce cortège, s'ajoutent l'Ours et le Renard dans le niveau attribué à l'Aurignacien ancien. La chair de la majorité de ces espèces ayant été prélevée, une acquisition via la chasse - et découlant des activités alimentaires - peut être proposée. Seuls l'Ours et le Mammouth n'entrent pas dans ce schéma. Les restes d'Ours sont abondants dans la grotte et la présence de plusieurs dents déciduales (dont certaines de chute) traduit une occupation d'une partie de la grotte des Abeilles comme tanière. Les dents d'Ours exploitées ont dès lors aisément pu être récoltées in situ. Dans le spectre faunique, le Mammouth est uniquement représenté par des fragments d'ivoire. L'ivoire frais étant très difficile à travailler (White 1997), sa présence aux Abeilles pourrait résulter d'un approvisionnement totalement disjoint de la sphère alimentaire. Pour les éléments d'industrie réalisés sur bois de cervidés, l'absence de base de bois ne permet pas de définir si la matière première utilisée relève d'une acquisition potentiellement en lien avec le gibier consommé (bois de massacre) ou si elle a été acquise en parallèle, par collecte.

Dans les deux ensembles, les éléments squelettiques utilisés comme supports correspondent à des fragments diaphysaires d'os longs de Bovinés, Cheval, Renne et Cerf et ont donc pu être directement puisés dans les déchets de boucherie alors disponibles. Leur récupération ne s'est toutefois pas faite de manière aléatoire puisque les supports utilisés présentent des dimensions importantes par rapport à la moyenne du corpus dans les deux ensembles. L'observation d'encoches de percussion multiples et alignées sur plusieurs retouchoirs de l'ensemble attribué à l'Aurignacien ancien pourrait par ailleurs indiquer que la sélection de certains supports s'est faite en amont des activités de récupération de la moelle osseuse. Les retouchoirs multiples sont également uniquement attestés dans l'ensemble attribué à l'Aurignacien ancien. Une sélection a également été opérée au niveau des espèces dans les deux ensembles, avec une prédilection pour les Bovinés, tel qu'observé dans d'autres séries du Paléolithique supérieur ancien (Soulier 2013). Cette préférence est très nettement perceptible dans le niveau attribué à l'Aurignacien ancien.

À l'instar de ce qui est décrit pour d'autres séries de l'Aurignacien ancien (Castel et Madelaine 2003 ; Castel, Chauvière, Madelaine 2003 ; Tartar 2009), deux canines d'Ours ont été exploitées comme support de retouchoir. En revanche, bien que plusieurs canines d'Ours aient été récoltées dans l'ensemble attribué au Protoaurignacien, aucune n'a été utilisée à cet escient. Des retouchoirs sur canine d'Ours existent toutefois dans d'autres séries relevant de ce technocomplexe (Soulier 2013 ; Soulier et al. 2014). La diversification des éléments squelettiques exploités à l'Aurignacien ancien a également concerné la mandibule, le radio-ulnaire et le métatarsien. Dans les deux ensembles, les côtes de grands ongulés ont été exploitées pour la réalisation de retouchoirs et, dans l'Aurignacien ancien, de lissoirs. Ces éléments squelettiques sont néanmoins très peu fréquents dans les corpus osseux récoltés ; des emports ponctuels, en vue de l'exploitation technique de ces supports, peuvent être proposés.

Les pièces transformées du niveau attribué à l'Aurignacien ancien attestent par ailleurs de la conduite d'activités plus diverses que dans le Protoaurignacien. Dans les deux ensembles, la présence de retouchoirs et de pièces intermédiaires atteste respectivement de la retouche/confection d'outils lithiques et d'activités de fendage. Les supports utilisés correspondant aux éléments les plus abondants dans les deux ensembles, leur fabrication a pu se dérouler aux Abeilles. En revanche, si les activités de chasse sont directement attestées par la présence de pointes de sagaie dans les deux ensembles, seul le niveau Aurignacien ancien a livré des déchets indiquant la confection d'au moins une partie de cet armement dans la grotte. Des éléments de parure ont uniquement été récoltés dans l'ensemble attribué à l'Aurignacien ancien ; la découverte d'une perle en panier inachevée parmi les restes fauniques (fig. 11) atteste de la confection d'une partie de la parure dans la grotte.

La présence de lissoirs et de poinçons - faisant directement référence à la préparation de peaux - est uniquement avérée dans l'ensemble attribué à l'Aurignacien ancien. Les saisons de capture identifiées pour le Renne, le Cheval et les Bovinés correspondent aux périodes où la peau est de qualité optimale (e.g. Ekblaw 1928 ; Soper 1941 ; Burch 1972 ; Binford 1978 ; Duncan

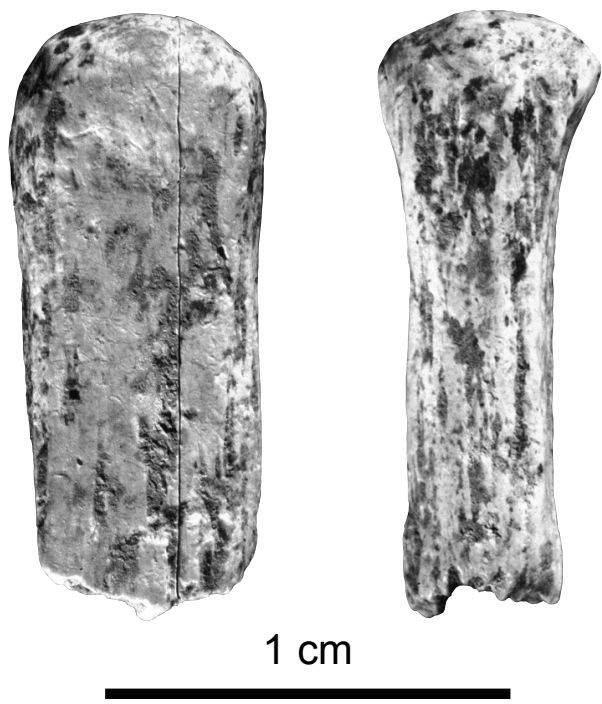

Figure 11 - Perle en panier en cours de fabrication découverte dans l'ensemble attribué à l'Aurignacien ancien des Abeilles (c) Musée National de Préhistoire.

Figure 11 - Unfinished basked-shaped bead from the Early Aurignacian assemblage of Les Abeilles (C) Musée National de Préhistoire. 
1992) et l'analyse des stries sur les restes fauniques offre un écho intéressant à cette activité. Les stries relatives au retrait de la peau sont en effet nombreuses dans les deux ensembles. Dans l'ensemble attribué à l'Aurignacien ancien, les incisions circulaires de la peau sont tout aussi bien localisées au niveau de l'acropode qu'en milieu de membre. Cette hétérogénéité pourrait indiquer que les groupes qui ont occupé le site des Abeilles à l'Aurignacien ancien n'ont pas toujours eu le même intérêt pour la peau (e.g. Wheat, Malde, Léopold 1972 ; Binford 1981 ; Grønnow, Meldgaard, Nielsen 1983). L'identification d'incisions longitudinales en face médiale des os du métapode indique néanmoins une volonté de récupérer des peaux de grandes dimensions. Par ailleurs, la multiplication des zones d'entame pourrait indiquer un retrait différé de la peau des bas-de-pattes, tel que pratiqué par de nombreux groupes humains vivant en contexte froid (e.g. Burch 1972 ; Binford 1981 ; Grønnow et al. 1983 ; Russell 1995). Dans le niveau attribué au Protoaurignacien, le retrait de la peau a été pratiqué sur une gamme d'espèces variée, et notamment sur de nombreux carnivores. La localisation des incisions circulaires d'entame, aux extrémités distales des pattes, témoigne du retrait des peaux dans leurs dimensions maximales. Le transport des fœtus peut également avoir été effectué en ce sens ; leur peau fine est en effet particulièrement prisée par de nombreux groupes de chasseurs-cueilleurs sub-actuels (Curtis 1930 ; Speck 1935 ; Blair 1966 ; Binford 1978, 1980, 1981 ; Speth 1983 ; Russell 1995 ; Nilssen 2000 ; Parget 2004 ; Peck 2004 ; Pinson 2004). Cet ensemble protoaurignacien n'a toutefois livré aucun outil clairement lié à la préparation de peaux mais on ne peut exclure un traitement postérieur, dans un autre lieu, ou avec des outils qui ne se sont pas conservés. Les tendons ont également été récupérés dans les deux ensembles. Si la peau et les tendons peuvent être consommés (e.g. Holston 1963 ; Burch 1972 ; Stopp 2000 ; Stefansson 2004 ; Costamagno et David 2009), leur utilisation la plus fréquente est d'ordre utilitaire (e.g. Fleming et Theodora 1979 ; Binford 1981 ; Russell 1995 ; Pasda 2013).

La dernière utilisation technique de la faune documentée aux Abeilles correspond à l'emploi de la matière osseuse comme combustible. D'après les critères proposés par Costamagno et collaborateurs (2009), la fréquence, taille et degré de combustion des éléments spongieux attestent de cette pratique dans l'ensemble attribué à l'Aurignacien ancien. Bien que des os brûlés soient présents dans l'ensemble attribué au Protoaurignacien, tous les critères ne sont pas réunis pour pouvoir proposer une utilisation de la graisse contenue dans les tissus spongieux comme combustible dans ce niveau.

En somme, la confrontation des données des deux ensembles illustre autant de similitudes que de différences. Le gibier exploité est de qualité optimale dans les deux ensembles et les choix de transport adoptés sont relativement similaires. Le nombre d'espèces exploitées à des fins alimentaires semble plus important dans l'ensemble attribué à l'Aurignacien ancien. Dans les deux ensembles, les stries de boucherie et les indices de percussion indiquent que les denrées alimentaires ont été quasi intégralement récupérées. Les modalités de désarticulation et les techniques de fracturation des ossements semblent toutefois avoir différé entre les deux techno-complexes. En parallèle de cette exploitation alimentaire, une utilisation de la faune à des fins techniques est documentée dans les deux ensembles, avec un emploi des bois de cervidés et des ossements pour la confection d'armes de chasse et d'outils domestiques. Une anticipation de cette exploitation technique de la faune pourrait avoir influé sur les saisons de capture du gibier puisque de nombreux éléments témoignent d'activités orientées autour du travail de peaux. En termes d'imbrication entre cette sphère technique et l'exploitation
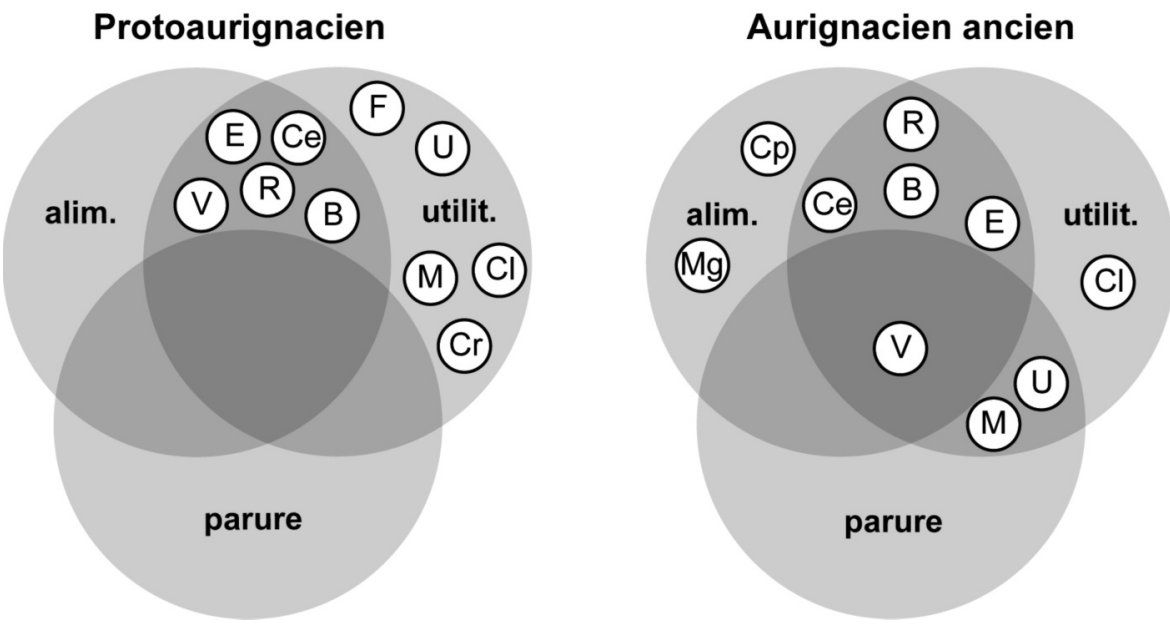

\author{
Légende : \\ E: Cheval, B: Bovinés, \\ R: Renne, Cp: Isard, \\ Ce: Cerf, Mg: Mégacéros \\ M: Mammouth, U: Ours, \\ V: Renard, Cl: Loup, \\ Cr: Hyène, $F$ : Chat
}

Figure 12 - Statut des espèces exploitées dans les ensembles aurignaciens des Abeilles. Abréviations : alim. = alimentaire ; utilit. = utilitaire.

Figure 12 - Status of the exploited species in the Aurignacian layers at les Abeilles. Abbreviations: alim. = food; utilit. = utilitarian. 
alimentaire, des différences importantes apparaissent entre les deux ensembles. La faune recueillie dans le niveau attribué au Protoaurignacien semble indiquer une intégration moins prononcée de l'exploitation technique de la faune dans les activités alimentaires ; plusieurs espèces sont uniquement exploitées à des fins techniques alors que dans l'ensemble attribué à l'Aurignacien ancien, les espèces intègrent généralement les différentes sphères d'activité (fig. 12). La chaîne opératoire technique semble avoir davantage interagi avec l'exploitation alimentaire du gibier dans l'ensemble Aurignacien ancien, dans le sens où elle paraît avoir dicté certains choix au moment de la prise de décision des éléments transportés jusque dans la grotte et lors des pratiques de boucherie. L'ensemble attribué au Protoaurignacien témoigne en revanche de la récupération de la peau d'un spectre d'espèces plus large, et notamment de peaux de carnivores ; un intérêt particulier pour les fœtus a également été observé dans cet ensemble. Dans les deux ensembles, l'exploitation technique de la matière animale apparaît néanmoins étroitement liée au domaine alimentaire.

\section{6 - Conclusion}

Les niveaux aurignaciens des Abeilles ont tous deux livrés d'abondants restes fauniques et leur étude documente plusieurs aspects de l'exploitation de la faune par les Hommes ayant occupé le site au Paléolithique supérieur ancien. L'intégration des restes fauniques transformés à l'étude archéozoologique a permis de montrer que l'exploitation du gibier est complexe dans les deux ensembles et répond à la fois à des besoins alimentaires et techniques.

Si des différences existent entre les deux ensembles, il est toutefois difficile de savoir si les "manques" observés dans l'ensemble attribué au Protoaurignacien par rapport à l'Aurignacien ancien sont réels ou s'ils sont artificiellement créés par la taille de l'échantillon et le moins bon état de conservation des vestiges fauniques de l'ensemble attribué au Protoaurignacien. À Isturitz, l'ensemble attribué au Protoaurignacien atteste d'une imbrication de ces différentes sphères toute aussi prononcée que ce qui est ici décrit pour l'Aurignacien ancien des Abeilles (Soulier 2013; Soulier et al. 2014). Ces différences ne sauraient dès lors être perçues comme une preuve de capacités - quelles qu'elles soient - moins développées des Hommes du Protoaurignacien par rapport à ceux de l'Aurignacien ancien et refléteraient davantage une diversité de réponses de ces groupes humains du Paléolithique supérieur ancien face à des besoins et/ou des contraintes différents.

\section{Remerciements}

Cette étude a bénéficié de l'appui financier du laboratoire TRACES-UMR 5608, du Ministère français de l'Enseignement supérieur et de la Recherche et du Social Sciences and Humanities Research Council du gouvernement du Canada (SSHRC) à travers le projet porté par E. Morin « Reassessing the Chronology, Composition and Climatic Implications of three Neandertal and Early Modern Human Sites from France ». Je tiens à remercier $M$. Crémilleux pour l'autorisation d'accès à la collection, ainsi que le Musée National de Préhistoire des Eyzies-de-Tayac où est hébergée la collection des Abeilles. Je tiens aussi à remercier le personnel du musée pour avoir contribué au bon déroulement de cette étude. Enfin, je souhaite remercier François Bon, Laura Eizenberg et Élise Tartar pour nos discussions qui ont grandement participé à une meilleure compréhension du gisement.

\section{Références bibliographiques}

ALTUNA J. 2006 - Faune de mammifères de la grotte préhistorique des Abeilles, Haute Garonne. In : LAPLACE G. et al. (eds.), Les Aurignaciens pyrénéens des Abeilles et méditerranéen de Régismont-le-Haut. Analyses typologique et paléontologique. Les Eyzies-de-Tayac: SAMRA, p. 142-153.

BACHELLERIE F. 2011 - Quelle unité pour le Châtelperronien ? Apport de l'analyse taphonomique et techno-économique des industries lithiques de trois gisements aquitains de plein-air : le Basté (PyrénéesAtlantiques), Bidart (Pyrénées-Atlantiques) et Canaule II (Dordogne). Thèse de doctorat, Université Bordeaux I, $441 \mathrm{p}$.

BARANDIARÁN I. 2006 - L'outillage aurignacien en os, bois de cervidé et ivoire de la grotte des Abeilles. In : LAPLACE G. et al. (eds.), Les Aurignaciens pyrénéens des Abeilles et méditerranéen de Régismont-le-Haut. Analyses typologique et paléontologique. Les Eyzies-de-Tayac: SAMRA, p. 112-123.

BARONE R. 1986 - Anatomie comparée des mammifères domestiques. Tome 1 - Ostéologie. Paris: Vigot, 761 p.

BEAUVAL C. et MORIN E. 2010 - Les repaires d'hyènes du Lussacois (Lussac-les-Châteaux, Vienne, France). Apport des sites des Plumettes et des Rochers-de-Villeneuve. In : BUISSON-CATIL J. et PRIMAULT J. (eds.), Préhistoire entre Vienne et Charente. Hommes et sociétés du Paléolithique. Villefranche-de-Rouergue: Ministère de la Culture et de la Communication, Mémoire 38 de l'Association des Publications Chauvinoises, p. 175-189.

BEZ J.-F. 1995 - Une expérience de découpe bouchère pratiquée au silex : aspects anatomiques. Préhistoire Anthropologie Méditerranéennes, 4, p. 41-50.

BIGNON O. 2006 - Approche morphométrique des dents jugales déciduales d'Equus caballus arcelini (sensu lato, Guadelli 1991) : critères de détermination et estimation de l'âge d'abattage. Compte Rendu Palevol, 5, p. 1005-1020.

BINFORD L.R. 1978 - Nunamiut Ethnoarchaeology. NewYork: Academic Press, 509 p.

BINFORD L.R. 1980 - Willow Smoke and Dogs' Tails: Hunter-Gatherer Settlement Systems and Archaeological Site Formation. American Antiquity, 45, 1, p. 4-20. 
BINFORD L.R. 1981 - Bones: Ancient Men and Modern Myths. Academic Press, 320 p.

BLAIR E.H. 1996 - The Indian Tribes of the Upper Mississippi Valley \& Region of the Great Lakes: As Described by Nicolas Perrot, French Commandant in the Northwest. University of Nebraska Press, $414 \mathrm{p}$.

BOCHERENS H., FIZET M. et MARIOTTI A. 1990 - Mise en évidence du régime alimentaire végétarien de l'ours des cavernes (Ursus spelaeus) par la biogéochimie isotopique $(13 \mathrm{C}, 15 \mathrm{~N})$ du collagène fossile. Compte Rendu de l'Académie des Sciences de Paris, 311, p. 1279-1284.

BON F. 2002 - L'Aurignacien entre Mer et Océan : Réflexion sur l'unité des phases anciennes de l'Aurignacien dans le sud de la France. Mémoire de la Société Préhistorique Française, $256 \mathrm{p}$.

BORDES J.-G. 2002 - Les interstratifications Châtelperronien/Aurignacien du Roc de Combe et du Piage (Lot, France) : analyse taphonomique des industries lithiques, implications archéologiques. Thèse de doctorat, Université de Bordeaux I, 365 p.

BRUGAL J.-P. et DAVID F. 1993 - Usure dentaire, courbe de mortalité et « saisonnalité »: les gisements du Paléolithique moyen à Grands Bovidés. In : DESSE J. et AUDOIN-ROUZEAU F. (eds.), Exploitation des animaux sauvages à travers le temps, Actes des XIII rencontres internationales d'Archéologie et d'Histoire d'Antibes. Juanles-Pins: APDCA, p. 64-67.

BUNN H.T., BARTRAM L.E. et KROLL E.M. 1988 Variability in Bone Assemblage Formation from Hadza Hunting, Scavenging, and Carcass Processing. Journal of Anthropological Archaeology, 7, p. 412-457.

BURCH E.S. 1972 - The Caribou/Wild Reindeer as a Human Resource. American Antiquity, 37, 3, p. 339-368.

CASTEL J.-C. 2011 - Archéozoologie de l'Aurignacien de l'Abri Castanet (Sergeac, Dordogne, France) : les fouilles 1994-1998. Revue de Paléobiologie, 30, 2, p.-783-815.

CASTEL J.-C. et MADELAINE S. 2003 - Stigmates observés sur les dents de grands carnivores à l'Aurignacien. L'exemple de l'Abri de La Souquette à Sergeac (Dordogne, France). Paleo, 15, p. 251-254.

CASTEL J.-C., CHAUVIĖRE F.-X. et MADELAINE S. 2003 - Sur os et sur dents : les « retouchoirs » aurignaciens de la Ferrassie (Savignac-de-Miremont, Dordogne). Paleo, 15 , p. $29-50$

CHURCH R.R. et LYMAN R.L. 2003 - Small fragments make small differences in efficiency when rendering grease from fractured artiodactyl bones by boiling. Journal of Archaeological Science, 30, 8, p. 1077-1084.

COSTAMAGNO S. 1999 - Stratégies de chasse et fonction des sites au Magdalénien dans le Sud de la France. Thèse de doctorat, Université de Bordeaux I, 495 p.
COSTAMAGNO S. et DAVID F. 2009 - Comparaison des pratiques bouchères et culinaires de différents groupes sibériens vivant de la renniculture. Archaeofauna, 19, p. 9-25.

COSTAMAGNO S., THÉRY-PARISOT I., CASTEL J.-C. et BRUGAL J.-P. 2009 - Combustible ou non ? Analyse multifactorielle et modèles explicatifs sur des ossements brûlés paléolithiques. In : THÉRY-PARISOT I., COSTAMAGNO S. et HENRY A. (eds.), Gestion des combustibles au Paléolithique et au Mésolithique : nouveaux outils, nouvelles interprétations. $\mathrm{XV}^{\mathrm{e}}$ Congrès de I'UISPP, septembre 2006, Lisbonne. BAR International Series, vol. 1914, p. 65-84.

CURTIS E.S. 1930 - The North American Indian. Weston La Barre, $409 p$.

DARWENT C.M. et LYMAN R.L. 2002 - Detecting the Postburial Fragmentation of Carpals, Tarsals, and Phalanges. In : HAGLUND W. D. et SORG M. H. (eds.), Advances in Forensic Taphonomy: Method, Theory, and Archaeological Perspectives. Boca Raton, FL.: CRC Press, p. 355-377.

DISCAMPS E. 2011 - Hommes et hyènes face aux recompositions des communautés d'Ongulés (MIS 5-3) : Éléments pour un cadre paléoécologique des sociétés du Paléolithique moyen et supérieur ancien d'Europe de l'Ouest. Thèse de doctorat, Université de Bordeaux I, $437 \mathrm{p}$.

DISCAMPS E., JAUBERT J. et BACHELLERIE F. 2011 Human choices and environmental constraints: deciphering the variability of large game procurement from Mousterian to Aurignacian times (MIS 5-3) in southwestern France. Quaternary Science Reviews, 30, p. 2755-2775.

DUNCAN P.B. 1992 - Horses and grasses: the nutritional ecology of equids and their impact on the Camargue. NewYork: Springer verlag, $287 \mathrm{p}$.

EIZENBERG L. 2006 - Le rôle de l'Aurignacien archaïque dans les phases initiales du Paléolithique supérieur: apports des industries lithiques de la grotte des Abeilles (Haute-Garonne). Mémoire de Master 2, Université de Toulouse-Le Mirail, 68 p.

EKBLAW W.E. 1928 - The Material Response of the Polar Eskimo to Their Far Arctic Environment (Continued). Annals of the Association of American Geographers, 18, 1, p. 1-24.

EMERSON A.M. 1990 - Archaeological Implications of variability in the economic anatomy of Bison bison. Thèse de doctorat, Washington State University, 896 p.

FISCHER J.W. 1995 - Bone Surface Modifications in Zooarchaeology. Journal of Achaeological Method and Theory, 2, 1, p. 7-68.

FLEMING R. et THEODORA H. 1979 - Ishi, the Last Yahi: A Documentary History. University of California Press, $256 \mathrm{p}$. 
FRISON G.C., WILSON M. et WILSON D.J. 1976 - Fossil bison and artifacts from an early Altithermal period arroyo trap in Wyoming. American Antiquity, 41, 1, p. 28-57.

GAMBIER D. 1992 - Vestiges humains du Paléolithique supérieur. Inventaire et description préliminaire de spécimens inédits des collections du Musée national de Préhistoire (Les Eyzies-de-Tayac). Paleo, 4, 91-100.

GRAYSON D.K. et DELPECH F. 2002 - Specialized Early Upper Palaeolithic Hunters in Southwestern France? Journal of Archaeological Science, 29, p. 1439-1449.

GRAYSON D.K. et DELPECH F. 2006 - Was there Increasing Dietary Specialization Across the Middle-toUpper Paleolithic Transition in France? In: CONARD N. (ed.) When Neanderthals and Modern Humans Met. Tübingen: Kerns Verlag, p. 377-417.

GRAYSON D.K., DELPECH F. et RIGAUD J.P. 2001 Explaining the Development of Dietary Dominance by a Single Ungulate Taxon at Grotte XVI, Dordogne, France. Journal of Archaeological Science, 28, p. 115-125.

GRØNNOW B., MELDGAARD M. et NIELSEN J.B. 1983 Aasivissuit, the Great Summer Camp: Archaeological, Ethnographical and Zoo-archaeological Studies of a Caribou-hunting Site in West Greenland. Man \& Society, $96 \mathrm{p}$.

HABERMEHL K.-H. 1975 - Die Altersbestimmung bei Haus- und Labortieren. Berlin-Hambur: Verlag Paul Parey, $216 \mathrm{p}$.

HOLSTON W.E. 1963 - The Diet of the Mountain Men. California Historical Society Quarterly, 42, 4, p. 301-309.

HOUSTON S., BALL T. et HOUSTON M. 2003 Eighteenth-Century Naturalists of Hudson Bay. McGillQueen's University Press, 432 p.

HUNGRY WOLF B. 1980 - The Ways of My Grandmothers. William Morrow \& Co, 256 p.

INGSTAD H. 1992 - The Land of Feast and Famine. McGillQueen's University Press, $360 \mathrm{p}$.

JENNESS D. 1923 - The Life of the Copper Eskimos. Report of the Canadian Arctic Expedition 1913-18, vol. XII, p. 277.

JIN J.J.H. et MILLS E.W. 2011 - Split phalanges from archaeological sites: Evidence of nutritional stress? Journal of Archaeological Science, 38, 8, p.1798-1809.

JULIEN M., BAFFIER D. et LIOLIOS D. 2002 - L'outillage en matières dures animales. In : SCHMIDER B. (ed.) L'Aurignacien de la grotte du Renne: Les fouilles d'André Leroi-Gourhan à Arcy-sur-Cure (Yonne). Paris: CNRS (XXXIVe suppl. à Gallia Préhistoire), p. 217-250.

KELSALL J.P. 1968 - The Caribou Ottawa: Canadian Wildlife Service, $340 \mathrm{p}$.
KENT S. 1993 - Variability in Faunal Assemblages: The Influence of Hunting Skill, Sharing, Dogs, and Mode of Cooking on Faunal Remains at a Sedentary Kalahari Community. Journal of Anthropological Archaeology, 12, 4, p. 323-385.

KNECHT H. 1991 - The Role of Innovation in changing Early Upper Paleolithic organic Projectile Technologies. Techniques et culture, 17, 8, p. 115-144.

KNECHT H. 1993 - Early Upper Paleolithic Approaches to Bone and Antler Projectile Technology. In: PETERKIN G. L., BRICKER N. M. et MELLARS P. (eds.), Hunting and Animal Exploitation in the Later Palaeolithic and Mesolithic of Eurasia. Special Issue (vol. 4, Issue 1). Archeological Papers of the American Anthropological Association, p. 33-47.

KREUTZER L.A. 1992 - Bison and Deer Bone Mineral Densities: Comparisons and Implications for the Interpretation of Archaeological Faunas. Journal of Archaeological Science, 19, p. 271-294.

LAM Y.M., CHEN X. et PEARSON O.M. 1999 Intertaxonomic Variability in Patterns of Bone Density and the Differential Representation of Bovid, Cervid, and Equid Elements in the Archaeological Record. American Antiquity, 64,2 , p. 343-362.

LAPLACE G. 1966 - Recherches sur l'origine et l'évolution des complexes leptolithiques. Paris: Ed. de Boccard, 586 p.

LAPLACE G. 1971 - De l'application des coordonnées cartésiennes à la fouille stratigraphique. Munibe, XXIII, p. 223-236.

LAPLACE G. et MÉROC L. 1954 - Application des coordonnées cartésiennes à la fouille d'un gisement. Bulletin de la Société préhistorique française, 51, 1-2, p. 58-66.

LAPLACE G., BARANDIARÁN I., SAENZ DE BURUAGA A. et ALTUNA J. 2006 - Les Aurignaciens pyrénéens des Abeilles et méditerranéen de Régismont-le-Haut, Analyses typologique et paléontologique. Les Eyzies-de-Tayac: SAMRA, $154 \mathrm{p}$.

LARIVIĖRE S. et PASITSCHNIAK-ARTS M. 1996 - Vulpes Vulpes. Mammalian Species, 537, p. 1-11.

LEECHMAN D. 1951 - Bone Grease. American Antiquity, 16, 4, p. 335-356.

LEROY-PROST C. 1974 - Les pointes en matière osseuse de l'Aurignacien. Caractéristiques morphologiques et essais de définition. Bulletin de la Société préhistorique française, 71, 2, p. 449-458.

LEROY-PROST C. 1975 - L'industrie osseuse aurignacienne. Essai régional de classification : Poitou, Charentes, Périgord. Gallia préhistoire, 18, 1, p. 65-156.

LEROY-PROST C. 1979 - L'industrie osseuse aurignacienne. Essai régional de classification : Poitou, Charentes, Périgord (suite). Gallia préhistoire, 22, 1, p. 205-370. 
LETOURNEUX C. 2003 - Devine qui est venu dîner à Brassempouy ? Approche taphonomique pour une interprétation archéozoologique des vestiges osseux de l'Aurignacien ancien de la grotte des Hyènes (Brassempouy, Landes). Thèse de Doctorat de l'Université de Paris I, $603 \mathrm{p}$.

LÉVINE M.A. 1979 - Archaeo-Zoological Analysis of Some Upper Pleistocene Horse Bone Assemblages in Western Europe. Thèse de doctorat, University of Cambridge, $372 \mathrm{p}$.

LIOLIOS D. 1999 - Variabilité et caractéristiques du travail des matières osseuses au début de l'Aurignacien: approche technologique et économique. Thèse de doctorat, Université de Paris X-Nanterre, 1999. $360 \mathrm{p}$.

LIOLIOS D. 2006 - Reflections on the role of bone tools in the definition of the Early Aurignacian. In: BAR-YOSEF O. et ZILHÃO J. (eds.), Towards a definition of the Aurignacian. Lisbonne: Instituto Poruguese de Arqueologia (Trabalhos de Arqueologia, 45), p. 37-51.

LUPO K.D. et SCHMITT D.N. 1997 - Experiments in bone boiling: nutritional returns and archaeological reflections. Anthropozoologica, 25-26, p. 137-144.

MALLYE J.-B. 2011 - Réflexion sur le dépouillement des petits carnivores en contexte archéologique : apport de l'expérimentation. Archaeofauna, 20, p. 7-25.

MAREAN C.W. et CLEGHORN N. 2003 - Large Mammal Skeletal Element Transport: Applying Foraging Theory in a complex Taphonomic System. Journal of Taphonomy, 1, 1, p. $15-42$.

MÉROC L. 1948 - Montmaurin (Coupe-Gorge, Montmaurin, Terrasse, Zubiate, les Abeilles). Informations préhistoriques. Xe circonscription. Gallia, VI, p. 409-412.

MÉROC L. 1963 - Les éléments de datation de la mandibule humaine de Montmaurin (Haute-Garonne). Bull. soc. géol. de France, V, p. 508-515.

METCALFE D. et JONES K.T. 1988 - A reconsideration of animal body part utility indices. American Antiquity, 53, 3, p. 486-504.

MILLER F.L. 1974 - Biology of the Kaminuriak population of barren-ground caribou. Part 2: Dentition as indicator of age and sex; composition and spezialization of the population. Canadian Wildlife Service Report Series, 31, 88 p.

MOREL P. et MÜLLER W. 1997 - HauteriveChampréveyres : un campement magdalénien au bord du lac de Neuchâtel. Étude archéozoologique. Neuchâtel: Archéologie neuchâteloise, $149 \mathrm{p}$.

MORIN E. 2007 - Fat composition and Nunamiut decisionmaking: a new look at the marrow and bone grease indices. Journal of Archaeological Science, 34, p. 69-82.
MORIN E. 2008 - Evidence for declines in human population densities during the early Upper Paleolithic in western Europe. PNAS, 105, 1, p. 48-53.

MORIN E. 2012 - Reassessing paleolithic subsistence: the Neandertal and modern human foragers of Saint-Césaire. Cambridge University Press, 358 p.

NILSSEN P.J. 2000 - An actualistic butchery study in South Africa and its implications for reconstructing hominid strategies of carcass acquisition and butchery in the upper Pleistocene and Plio-Pleistocene. Thèse de doctorat, University of Cape Town, $649 \mathrm{p}$.

NORMAND C. 2005 - Les occupations aurignaciennes de la grotte d'Isturitz (Saint-Martin-d'Arberoue ; PyrénéesAtlantiques ; France) : synthèse des données actuelles. Munibe (Antropologia-Arkeologia), 57, Homenaje a Jesús Altuna, p. 119-129.

OUTRAM A. et ROWLEY-CONWY P. 1998 - Meat and marrow utility indices for horse (Equus). Journal of Archaeological Science, 25, p. 839-849.

OUTRAM A.K. 2001 - A New Approach to Identifying Bone Marrow and Grease Exploitation: Why the "Indeterminate" Fragments should not be Ignored. Journal of Archaeological Science, 28, p. 401-410.

OUTRAM A.K. 2002 - Bone Fracture and Within-Bone Nutrients: An Experimentally Based Method for Investigating Levels of Marrow Extraction. In: MIRACLE P. et MILNER N. (eds.), Consuming Passions and Patterns of Consumption. Cambridge: McDonald Institute for Archaeological Research, p. 51-64.

OUTRAM A.K. 2005 - Distinguishing bone fat exploitation from other taphonomic what the high level of bone fragmentation at the Middle Neolithic site of Ajvide, Gotland? In: MULVILLE J. et OUTRAM A. (eds.), The zooarchaeology of fats, oils, milk and dairying. Oxford: Oxbow Books, p. 32-43.

PARGET A.M. 2004 - The People of the Plains. University of Regina. Canadian Plains Research Center, 78 p.

PASDA K. 2013 - Caribou hunting and utilization in West Greenland: Past and present variants. Anthropozoologica, 48, 1, p. 111-123.

PECK T.E. 2004 - Bison Ethology and Native Settlement Patterns during the Old Women's Phase on the Northwestern Plains. BAR Series 1278, 147 p.

PELEGRIN J. 1990 - Observations technologiques sur quelques séries du Châtelperronien et du MTA B du sudouest de la France. Une hypothèse d'évolution. In: FARIZY C. (ed.) Paléolithique moyen récent et Paléolithique supérieur ancien en Europe, actes du colloque international de Nemours. Mémoire du Musée de Préhistoire d'lle-de-France, p. 195-201. 
PINSON E.B. 2004 - Alaska's Daughter. An Eskimo Memoir of the Early Twentieth Century. Logan: Utah State University Press, $212 \mathrm{p}$.

ROINE K., NIEMINEN M. et TIMISJÄVI J. 1982 - Foetal Growth in the Reindeer. Acta vet. scand., 23, p. 107-117.

ROUSSEL M. 2011 - Normes et variations de la production lithique durant le Châtelperronien : la séquence de la Grande-roche-de-la-Plématrie à quinçay (Vienne). Thèse de doctorat, Université de Paris Ouest Nanterre, 540 p.

RUSSELL P.N. 1995 - Some Large Game Animal Traditions of the Inland Dena'Ina. University of Alaska, $15 \mathrm{p}$.

SAINT-GERMAIN C. 2005 - Animal fat in the cultural world of the Native Peoples of Northeastern America. In: MULVILLE J. et OUTRAM A. (eds.), The zooarchaeology of fats, oils, milk and dairying. Oxford: Oxbow Books, p. 107-113.

SEKHR A. 1998 - Étude archéozoologique des niveaux aurignaciens (couches 14 à 6) et de la base des niveaux gravettiens (niveaux X à T4) de l'abri Pataud (Les Eyzies, Dordogne). Thèse de doctorat, Muséum National d'Histoire Naturelle de Paris, $346 p$.

SIMONNET R. 1979 - Les habitats préhistoriques des gorges de la Save dans le massif de LespugueMontmaurin. In : SACCHI D. (ed.) Le Pléistocène supérieur en France : Ille colloque Franco-Soviétique Dynamique des interactions entre le milieu naturel et les sociétés préhistoriques, 10-25 septembre 1979. p. 155-162.

SOPER J.D. 1941 - History, Range, and Home Life of the Northern Bison. Ecological Monographs, 11, 4, p. 347-412.

SOULIER M.-C. 2013 - Entre alimentaire et technique : l'exploitation animale aux débuts du Paléolithique supérieur. Stratégies de subsistance et chaînes opératoires de traitement du gibier à Isturitz, La Quina aval, Roc-deCombe et Les Abeilles. Thèse de doctorat, Université de Toulouse-Le Mirail, 754 p.

SOULIER M.-C., GOUTAS N., NORMAND C., LEGRAND A. et WHITE R. 2014 - Regards croisés de l'archéozoologue et du technologue sur l'exploitation des ressources animales à l'Aurignacien archaïque : l'exemple d'Isturitz (Pyrénées-Atlantiques, France). In : THIÉBAULT C., CLAUD É. et COSTAMAGNO S. (eds.), Transitions, ruptures et continuité en Préhistoire. Vol.2 : Exploitation des ressources organiques à la fin du Paléolithique moyen et au début du Paléolithique supérieur : interactions entre environnement et comportements techniques. XXVII congrès préhistorique de France, Bordeaux-Les Eyzies, 31 mai-5 juin 2010: Mémoires de la SPF, p. 315-332.

SPECK F.G. 1935 - Naskapi: The Savage Hunters of the Labrador Peninsula. University of Oklahoma Press, 257 p.

SPETH J.D. 1983 - Bison Kills and Bone Counts: Decision Making by Ancient Hunters. University of Chicago Press, $272 \mathrm{p}$.
STEFANSSON V. 2004 (rééd. 1913) - My Life With The Eskimo. Kessinger Publishing, $672 \mathrm{p}$.

STOPP M.P. 2000 - Après la capture des phoques et des caribous. Une reformulation des modèles d'adaptation dans le Subarctique oriental. Recherches amérindiennes au Québec, 2, p. 51-62.

TABORIN Y. 1990 - Les prémices de la parure. In: FARIZY C. (ed.) Paléolithique moyen récent et Paléolithique supérieur ancien en Europe. Mémoire du Musée de Préhistoire d'lle-de-France (3), p. 335-344.

TABORIN Y. 2002 - Les objets de parure. In: SCHMIDER B. (ed.) L'Aurignacien de la Grotte-du-Renne, XXXIV supplément à Gallia Préhistoire. CNRS Éditions, p. 253-256.

TARTAR É. 2009 - De l'os à l'outil. Caractérisation technique, économique et sociale de l'utilisation de l'os à l'Aurignacien ancien. Étude de trois sites : l'Abri Castanet (secteurs nord et sud), Brassempouy (Grotte des Hyènes et Abri Dubalen) et Gatzarria. Thèse de doctorat, Université de Paris I - Panthéon Sorbonne, 375 p.

TEYSSANDIER N. 2007 - En route vers l'Ouest. Les débuts de l'Aurignacien en Europe. BAR International Series 1638, $312 \mathrm{p}$.

THIÉBAUT C., CLAUD E., COSTAMAGNO S., COUDENNEAU A., DESCHAMPS M., MOURRE V., VAL A., BRENET M., CHACÓN M.-G., CHONG S., COUMONT M.-P., COLONGE D., GERBE M., MUSSINI C. et SOULIER M.-C. 2011 - Des traces et des hommes : Projet de recherche interdisciplinaire sur l'identification des modalités d'acquisition et de traitement des matières végétales et animales au Paléolithique moyen en Europe occidentale. Rapport. SRA Midi-Pyrénées, 222 p.

VANHAEREN M. 2002 - Les fonctions de la parure au Paléolithique supérieur : de l'individu à l'unité culturelle. Thèse de doctorat, Université de Bordeaux I, 355p.

VIGNE J.-D. 2005 - Découpe du cerf (Cervus elaphus) au Mésolithique moyen, à Noyen-sur-Seine (Seine-et-Marne) : analyses tracéologique et expérimentale. Revue de Paléobiologie, 10, p. 69-82.

VIGNE J.-D., MARINVAL-VIGNE M.C., LANFRANCHI F.D. et WEISS M.C. 1981 - Consommation du « Lapin-rat » (Prolagus sardus Wagner) au Néolithique ancien méditerranéen. Abri d'Araguina-Sennola (Bonifacio, Corse). Société Préhistorique Française, 78, p. 222-224.

VILLA P. et MAHIEU E. 1991 - Breakage patterns of human long bones. Journal of Human Evolution, 21, 1, p. 27-48.

VILLA P., CASTEL J.-C., BEAUVAL C., BOURDILLAT C. et GOLDBERG P. 2004 - Human and carnivore sites in the European Middle and Upper Paleolithic: similarities and differences in bone modification and fragmentation. Revue de Paléobiologie, 23, 2, p. 705-730. 
WHEAT J.B., MALDE H.E. et LEOPOLD E.B. 1972 - The Olsen-Chubbuck Site: A Paleo-Indian Bison Kill. Society for American Archaeology, $180 \mathrm{p}$.

WHITE R. 1997 - Substantial acts: From materials to meaning in Upper Paleolithic representation. In: CONKEY M., SOFFER O., STRATMANN D. et JABLONSKI N. G. (eds.), Beyond Art: Pleistocene Image and Symbol. San Francisco: Memoirs of the California Academy of Sciences, 23, p. 93-121.

WHITE R. 2002 - Observations technologiques sur les objets de parure. In : SCHMIDER B. (ed.) L'Aurignacien de la grotte du Renne. Les fouilles d'André Leroi-Gourhan à Arcy sur Cure (Yonne). Paris: XXXIVe supplément à Gallia Préhistoire. CNRS éditions, p. 257-266.
WHITE R. 2010 - Les parures de l'Aurignacien ancien et archaïque. Perspectives technologiques et régionales des fouilles récentes. In : MISTROT V. (ed.) De Néandertal à l'Homme moderne : l'Aquitaine préhistorique, vingt ans de découvertes (1990-2010). Bordeaux: Editions Confluences, p. 93-103. 
